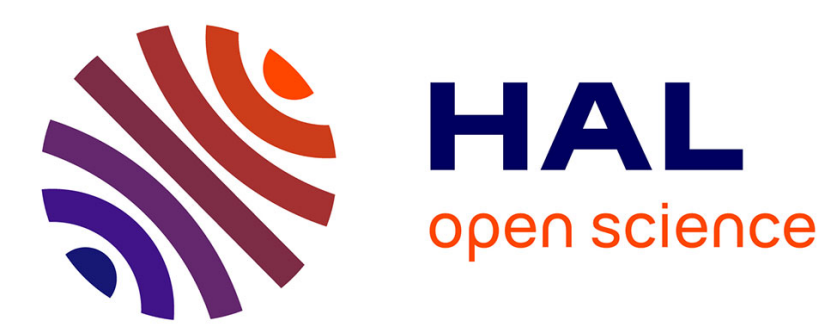

\title{
A flexible route to new spirodioxanes, oxathianes and morpholines
}

Marlène Goubert, Isabelle Canet, M.-E. Sinibaldi

\section{To cite this version:}

Marlène Goubert, Isabelle Canet, M.-E. Sinibaldi. A flexible route to new spirodioxanes, oxathianes and morpholines. Tetrahedron, 2009, 65, pp.4182-4189. hal-00378071

\section{HAL Id: hal-00378071 \\ https://hal.science/hal-00378071}

Submitted on 23 Apr 2009

HAL is a multi-disciplinary open access archive for the deposit and dissemination of scientific research documents, whether they are published or not. The documents may come from teaching and research institutions in France or abroad, or from public or private research centers.
L'archive ouverte pluridisciplinaire HAL, est destinée au dépôt et à la diffusion de documents scientifiques de niveau recherche, publiés ou non, émanant des établissements d'enseignement et de recherche français ou étrangers, des laboratoires publics ou privés. 


\title{
A flexible route to new spirodioxanes, oxathianes, and morpholines
}

\author{
Marlène Goubert, Isabelle Canet, and Marie-Eve Sinibaldi
}

Clermont Université, Université Blaise Pascal, CNRS UMR 6504, SEESIB 63177 Aubière Cedex, France

\begin{abstract}
This work describes a modular efficient route to 10-aza-4-thia-, 10-aza-4-oxa-, and 10-oxa-4-thia-1,7dioxaspiro[5.5] undecanes. The synthetic pathway relies upon the iterative nucleophilic substitution of 1,3-dichloropropan-2-one $O$-benzyloxime by solketal derivatives. The oxime key-intermediates, submitted to an acidic deprotection-spiroacetalization process, afforded these original spiroketal compounds in three steps, few purifications, and very good yields.
\end{abstract}

\section{Introduction}

Spiroketals are found as crucial substructures in a wide variety of natural compounds of diverse origins. ${ }^{[1]}$, [1a], [1b], [1c] and [1d] Due to their broad spectrum of biological activities, these moieties have therefore attracted considerable synthetic interest leading to numerous strategies, which have been described and

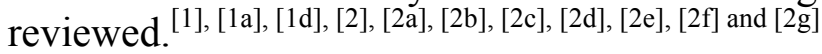

Recently, it has been recognized that for spiroketal-containing drug compounds, the incorporation of a heteroatom at the C-4 position in one cycle of the spiranic core led to an enhancement of their activity. Thus, a series of new spiroketal derivatives possessing a nitrogen atom were evaluated as tachykinin antagonists; these spiromorpholino compounds showed a high affinity and excellent CNS penetration. ${ }^{[3],}$ $[3 \mathrm{a}]$ and ${ }^{[3 \mathrm{~b}]}$ By the same way, preparation of aza- ${ }^{[4] \text { and }[4 \mathrm{~b}]}$ or thia- ${ }^{5}$ analogues of the $\mathrm{GM}_{3}$ ganglioside lactone led to useful compounds that revealed hydrolytically stable immunogens. Lastly, the modification of the functionality of the tonghaosu skeleton afforded new substrates showing obvious antifeedant activity. ${ }^{[6] \text {, }}$ [6a] and [6b]

The main approach for the synthesis of spiroketal units involves (i) elaboration of a key acyclic keto-diol or its equivalent ${ }^{[7],[7 \mathrm{a}], \text { [7b], [7c], [7d], [7e], [7f], [7g] and [7h] }}$ via intermolecular $\mathrm{C}-\mathrm{C}$ bond formations followed by (ii) an intramolecular acid-catalyzed dehydrative ketalization. In this approach, the control of the stereochemistry of the anomeric center is usually based on the relative stabilities of the different isomers in the spiroketalization process: when maximum anomeric effects and minimum steric interactions are conjugated, a thermodynamically most favored isomer is formed as the almost sole product. However, although this strategy has been largely reported for the synthesis of numerous spiroketal skeletons, it has been rarely used for the elaboration of diheteroatom-containing spiroketals.

Indeed, and to our knowledge, only two syntheses of 1,7-dioxaspiro[5.5] undecanes incorporating heteroatoms at the C-4 and C-10 positions were reported (Scheme 1). The first one rested on the use of a d-fructose unit as chiral source. ${ }^{[8],[8 \mathrm{a}] \text { and }[8 \mathrm{~b}]}$ The authors employed a step-by-step nucleophilic substitution by chloroethanol followed by an oxidative fructose-ring opening to prepare the key intermediate 1, from which the 10-thia- or the 10-aza-1,4,7-trioxaspiro[5.5] undecanes $\mathbf{2}$ and $\mathbf{3}$ were elaborated, once again, through nucleophilic displacements. The targeted spiroketals were thus isolated as sole isomers, in seven steps from fructose in 19\% and 33\% overall yields, respectively. This approach has not been extended to substituted derivatives, probably because of the choice of the starting material. 

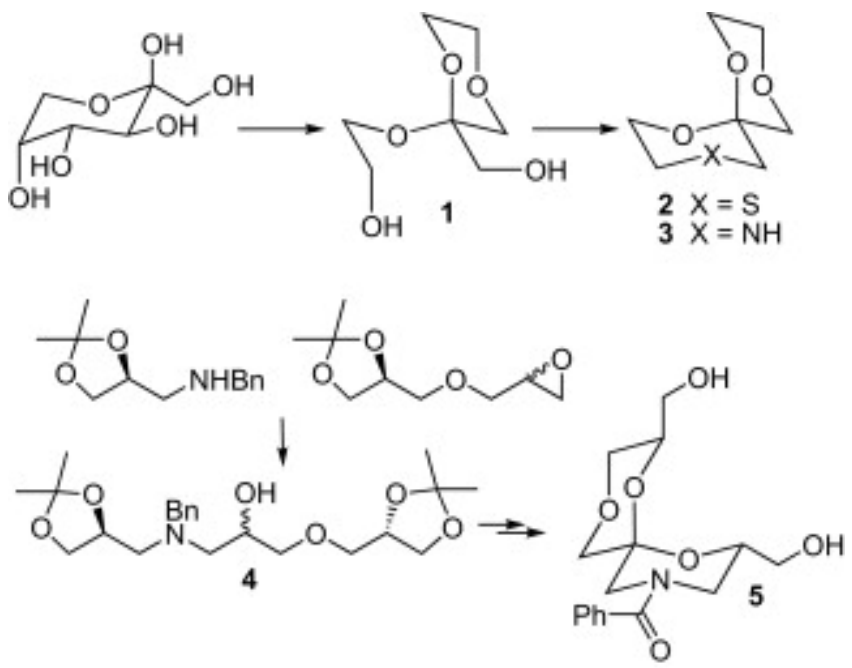

Scheme 1.

The second method ${ }^{8 c}$ consisted of the regioselective ring opening of the 2,2-dimethyl-4-[(oxiran-2ylmethoxy)methyl]-1,3-dioxolane (obtained from solketal and epichlorohydrin) by the (2,2-dimethyl-1,3dioxolan-4-yl)methanamine, leading to the key alcohol 4. Finally, oxidation and acid-catalyzed deprotection-spiroketalization conduced to the 10-aza-1,4,7-trioxaspiro[5.5] undecane 5 (seven steps, $22 \%$ overall yield) but only after modification of the nitrogen protective group. The use of an oxidation step in this synthetic scheme disfavored the direct introduction of a sulfur atom at C-4.

For our part, we have recently demonstrated' that the synthesis of 'symmetrical' new 1,4,7,10tetraoxaspiro[5.5] undecane $\mathbf{6 a}$ and 4,10-dithia-1,7-dioxaspiro[5.5] undecane $\mathbf{6 b}$ could be efficiently achieved through a one-step acid-catalyzed deprotection-spiroketalization from the elaborated key oximes 7a,b; these latter being readily synthesized by a one-step double substitution of 1,3dichloropropan-2-one $O$-benzyloxime $\mathbf{8}$ by solketal 9a or its thiol derivative 9b (Scheme 2). Disappointingly, this synthetic pathway revealed ineffective to elaborate the biologically interesting 4,10aza-1,7-dioxaspiro[5.5] undecane framework 6c and led to the 3-aza-6,8-dioxabicyclo[3.2.1]octan-2-one 10 in place of the spiranic system (Scheme 2). With oxime 7c, the presence of amine functions made critical the final spirocyclization and gave numerous by-products. ${ }^{10}$

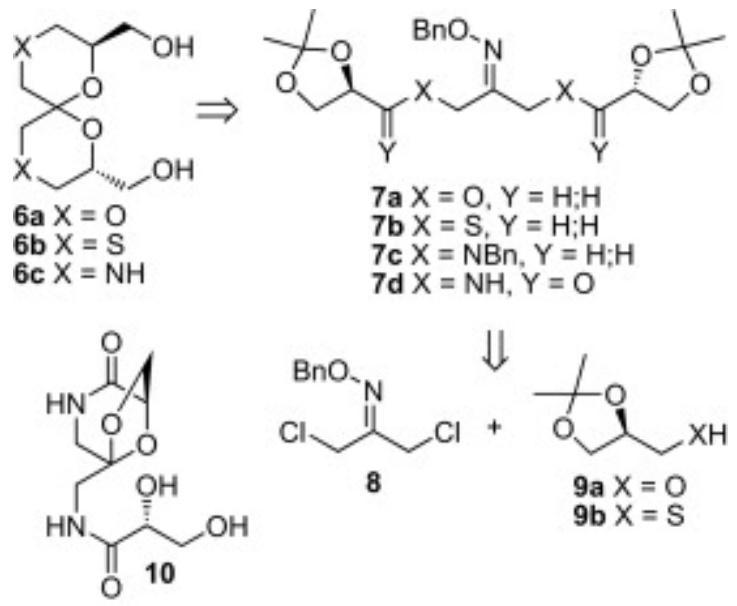

Scheme 2 .

Nevertheless, and even if spirobismorpholines could not be obtained, we pursued the study of this strategy to elaborate this time 'dissymmetrical' spiroketals possessing different heteroatoms in C-4 and C10 positions. In this paper, we would like to present our results toward the successful syntheses of three families of original compounds owning a 2,8-dihydroxymethyl-4-thia-1,7,10-trioxaspiro[5.5] undecane, a 2,8-dihydroxymethyl-10-aza-4-thia-1,7-dioxaspiro[5.5]undecane, and a 2,8-dihydroxymethyl-10-aza1,4,7-trioxaspiro[5.5] undecane skeleton.

\section{Results and discussion}


The crucial point for the elaboration of these spiroketals was the efficient control of the monosubstitution of 1,3-dichloropropan-2-one $O$-benzyloxime 8 by solketal derivatives 9 .

In the conditions used for oxa- and thia-series $\mathbf{6 a}, \mathbf{b}$ - alkali hydrides treatment - mono-substituted oximes were detected during the TLC monitoring as the first product of the reaction but usually very fast accompanied with the disubstituted compounds. ${ }^{9}$ We therefore undertook a study of the best operating conditions in terms of base, concentration ratio, solvent, and temperature to obtain oximes 11a,b from 8 and 9a,b and avoid the more favored double substitution. In all cases, reactions were TLC-monitored and quenched at the appearance of disubstituted oximes 7. From alkali hydrides, best results were obtained by carrying out the reaction in tetrahydrofuran $\left(1 \mathrm{mmol}\right.$ of $8 / 3 \mathrm{~mL}$ of solvent) at $20^{\circ} \mathrm{C}$, with an addition of 1 equiv of alcohol 9a or freshly prepared thiol $\mathbf{9 b}$ on a mixture of oxime $\mathbf{8}$ and 2 equiv of alkali hydride. These conditions led to the expected chlorides 11a and 11b in $30 \%$ and $64 \%$ yields, respectively, accompanied with only $5-10 \%$ of the corresponding undesired $b i$-adduct $7 \mathbf{a}$ or $7 \mathbf{b}$. Unreacted starting materials 9a and 9b could be recycled from flash column chromatography. In the case of 11a, better yields - even if more wavering, from $40 \%$ to $60 \%$ yield - were obtained using a similar procedure as that previously described for the synthesis of 4-hydroxymethyl-1,3-dioxolane ethers: ${ }^{11}$ namely, treatment of solketal by potassium hydroxide in toluene at $70{ }^{\circ} \mathrm{C}$ followed by the slow addition of an excess (1.5 equiv) of oxime 8 (Scheme 3 ).

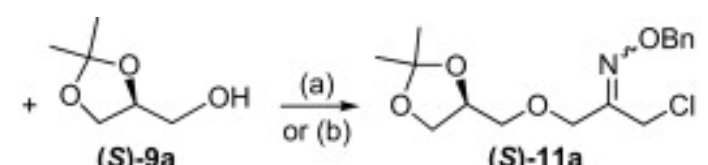

(S)-9a

(S)-11a

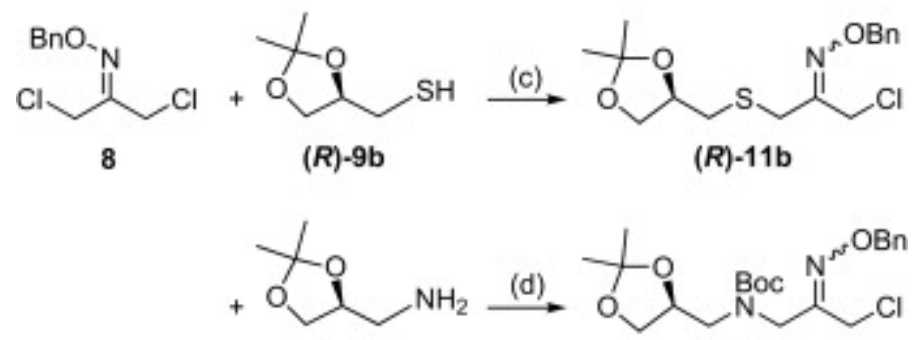

(S)-9c

(S)-11c

Scheme 3. Reagents and conditions: (a) KH (2 equiv), THF, $20{ }^{\circ} \mathrm{C}, 30 \%$; (b) KOH (1 equiv), $18-\mathrm{C}-6$ ( 0.5 equiv), toluene, $70{ }^{\circ} \mathrm{C}, 40-60 \%$; (c) $\mathrm{NaH}$ (2 equiv), THF, $20{ }^{\circ} \mathrm{C}, 64 \%$; (d) two steps, $28 \%$ overall yield. ${ }^{10}$

Compound 11a appeared as a mixture of $Z$ and $E$ isomers in a $1 / 1$ ratio determined from the ${ }^{1} \mathrm{H}$ NMR spectrum, whereas compound 11b was a mixture of the two isomers in a $3 / 2$ ratio in favor of the $Z$ one. ${ }^{12}$ Concerning the synthesis of 'aza-monooximes', our exploratory work in this $\operatorname{area}^{10}$ has shown that nitrogen atoms implied in amine function (i.e., oxime 7c, Scheme 2) hindered the final spiroketalization carried out under acidic conditions. Furthermore, the extensive study toward aza-substitution of oxime 8 allowed us to prepare the monochlorooxime 11c ${ }^{10}$ issued from 8 and the Boc-derivative of $9 \mathbf{c}$ in a correct yield of 33\% (Scheme 3). Consequently, this kind substrate had also been considered for the synthesis of new 'dissymmetrical' 4-aza-1,7-dioxaspiro[5.5] undecanes.

With the mono-substituted chlorooximes 11a-c in hands, we engaged them in spiroketal synthesis. Considering our previous results in the symmetrical series, we first explored the access to 2,8dihydroxymethyl-4-thia-1,7,10-trioxaspiro[5.5] undecane 13. This was realized according to two parallel pathways using both 11a and 11b as key building blocks (Scheme 4). Thus, conversion of 11a,b to the required oxime 12 was achieved using $\mathrm{KH}$ in tetrahydrofuran at $20^{\circ} \mathrm{C}$, and gave, after purification, oxime 12 in 86\% yield (from 11a and $7 \mathbf{b}$ ) and 54\% yield (from 11b and 7a), respectively. Compound 12 appeared as a mixture of $Z$ and $E$ isomers ( $3 / 2$ ratio determined on ${ }^{1} \mathrm{H}$ NMR spectrum) easily identified by the chemical shift of the methylene group in the $\alpha$ position of the benzyloxime function. ${ }^{12}$ Lastly, oxime 12 was heated under reflux for 2 days in an acetone/water solution in the presence of Amberlyst ${ }^{\circledR}$ 15. In this case and as in 4,10-dithia-1,7-dioxaspiro[5.5] undecane series, the addition of 10 equiv of paraformaldehyde was necessary to achieve efficiently the ketone deprotection and promote the spiroketalization (Scheme 4). Spiroketal 13 was thus obtained in $79 \%$ yield as a mixture of two inseparable isomers $\mathbf{1 3 a} / \mathbf{1 3 b}$ in a $19 / 1$ ratio as determined on the quantitative ${ }^{13} \mathrm{C}$ NMR spectrum. 


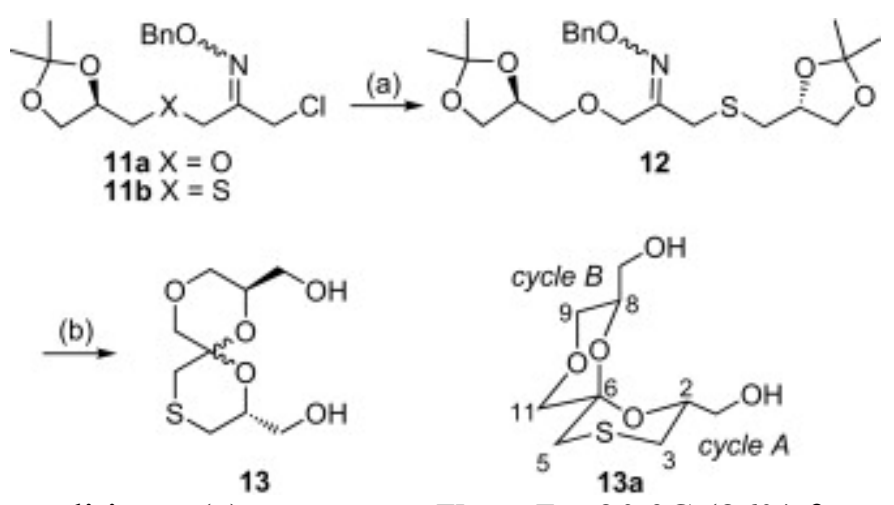

Scheme 4. Reagents and conditions: (a) KH, THF, $7 \mathbf{b}$ or $7 \mathbf{a}, 20{ }^{\circ} \mathrm{C}(86 \%$ from 11a, $54 \%$ from 11b); (b) Amberlyst ${ }^{\circledR} 15,\left(\mathrm{CH}_{2} \mathrm{O}\right)_{n}$, acetone $/ \mathrm{H}_{2} \mathrm{O}(10: 1), \Delta, 48 \mathrm{~h}, 79 \%$.

This result was in agreement with those observed in the symmetrical series. ${ }^{9}$ Indeed, in our spirocyclisation acid-catalyzed conditions, dioxa-spiroketal 6a (Scheme 1) was isolated as a sole isomer, possessing a $C 2$ symmetry with a double anomeric effect, having two cycles in a chair conformation and the hydroxymethyl groups in an equatorial position. Conversely, we observed for dithia-spiroketal $\mathbf{6 b}$ a loss of stereoselectivity due to the presence of the less electron-withdrawing sulfur atom. Thus, starting from enantiopure solketal 9a, we isolated a mixture of two C-6 epimers.

The major isomer 13a could be isolated and characterized after a classical TBDPSprotection/deprotection sequence. Its ${ }^{13} \mathrm{C}$ NMR spectrum comprised nine peaks with a spiranic carbon detected at $92.3 \mathrm{ppm}$ (see Table 1). In the ${ }^{1} \mathrm{H}$ NMR spectrum, $\mathrm{H}-2(\delta=4.06 \mathrm{ppm})$ and $\mathrm{H}-8(\delta=4.02 \mathrm{ppm})$ exhibited vicinal coupling constants ${ }^{3} J$ with $\mathrm{H}-3$ and $\mathrm{H}-9$ of 11.0 and $2.0 \mathrm{~Hz}, 11.0$ and $2.5 \mathrm{~Hz}$, respectively. Thus, 13a adopted a chair conformation for its two cycles with $\mathrm{H}-2$ and $\mathrm{H}-8$ in an axial position. Moreover, structural data for 13a fitted with those of (6S)-2,8-dihydroxymethyl-4,10-dithia-1,7dioxaspiro[5.5] undecane $\mathbf{6 b}$ for its $\mathrm{A}$ cycle and (6S)-2,8-dihydroxymethyl-1,4,7,10tetraoxaspiro[5.5] undecane 6a for its B cycle (see Table 1): for instance, C-3 was found at 27.5 ppm and C-9 at $68.7 \mathrm{ppm}$. The values of the chemical shifts of C-2 and C-8 in 13a, are also similar to those observed in the symmetrical series. Because of the oxathiane-dioxane sequence, the chemical shifts of C5 and C-11 were slightly modified and were observed, now, at $31.0 \mathrm{ppm}$ and $72.9 \mathrm{ppm}$, respectively. Their corresponding hydrogens were shielded for H-5 and deshielded for H-11.

Table 1.

NMR data for 'symmetrical' and 'dissymmetrical' spirobisdioxanes $\mathbf{6 a},{ }^{9}$ spirobisoxathianes $\mathbf{6 b},{ }^{9}$ and spirodioxane-oxathiane 13a (recorded at $400 \mathrm{MHz}$, measured in $\mathrm{CD}_{3} \mathrm{OD}$ )

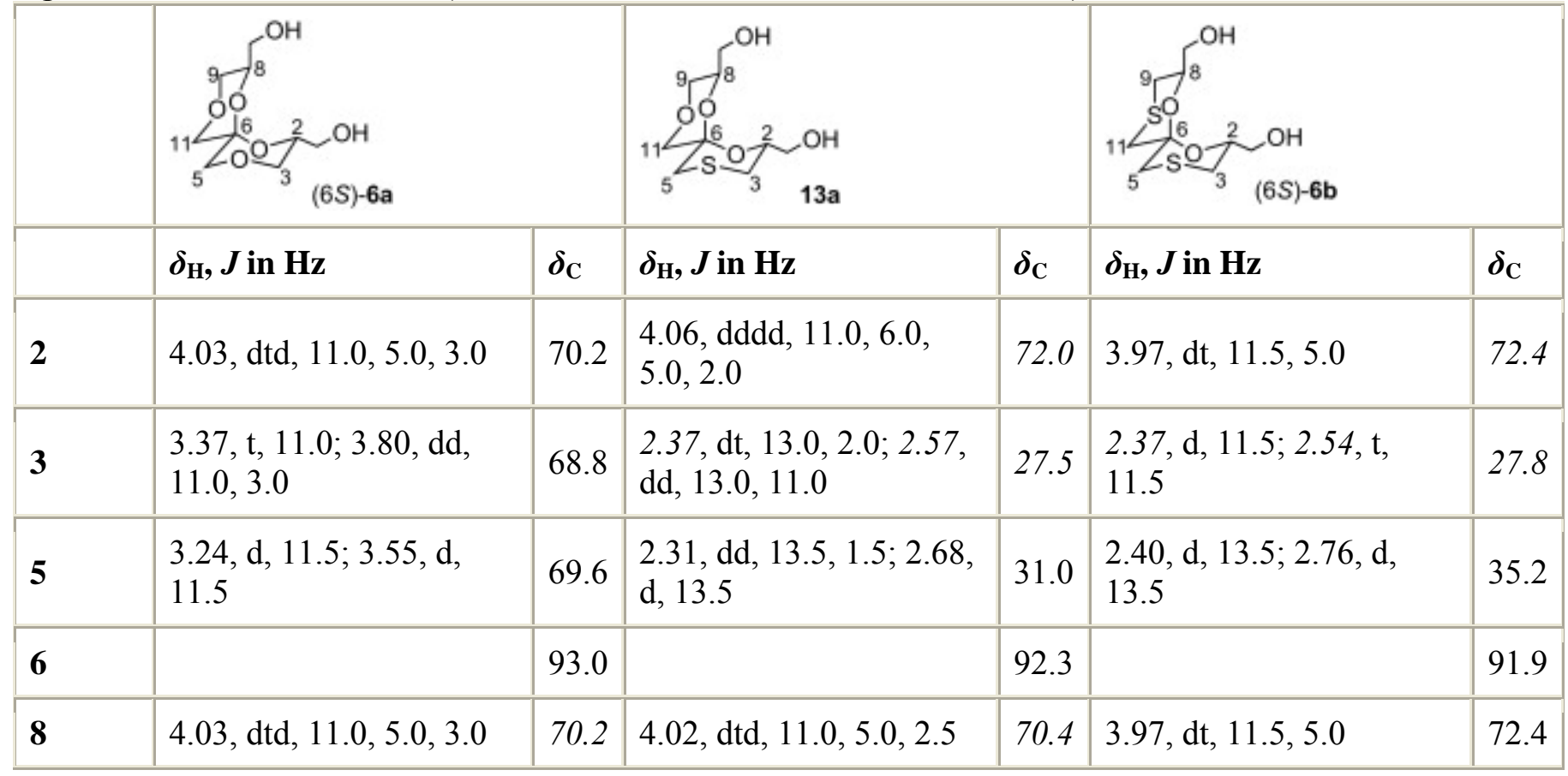




\begin{tabular}{|c|c|c|c|c|c|c|}
\hline & $\int_{5}^{9} \int_{36}^{\mathrm{OH}}{ }_{3}^{2}$ & & 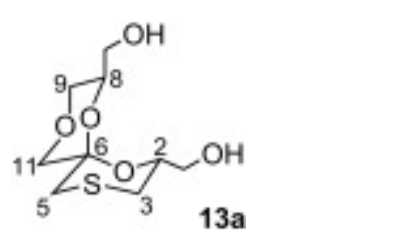 & & $\underbrace{{ }_{\mathrm{S}}^{\mathrm{O}}-\overbrace{3}^{2}}_{5}$ & \\
\hline & $\delta_{\mathrm{H}}, J$ in $\mathrm{Hz}$ & $\delta_{\mathrm{C}}$ & $\delta_{\mathrm{H}}, J$ in $\mathrm{Hz}$ & $\delta_{\mathrm{C}}$ & $\delta_{\mathrm{H}}, J$ in $\mathrm{Hz}$ & $\delta_{\mathrm{C}}$ \\
\hline 9 & $\begin{array}{l}3.37, \mathrm{t}, 11.0 ; 3.80, \mathrm{dd}, \\
11.0,3.0\end{array}$ & 68.8 & $\begin{array}{l}3.41, \mathrm{t}, 11.0 ; 3.84, \mathrm{dd}, \\
11.5,2.5\end{array}$ & 68.7 & $\begin{array}{l}2.37, \mathrm{~d}, 11.5 ; 2.54, \mathrm{t}, \\
11.5\end{array}$ & 27.8 \\
\hline 11 & $\begin{array}{l}3.24, \mathrm{~d}, 11.5 ; 3.55, \mathrm{~d} \\
11.5\end{array}$ & 69.6 & $\begin{array}{l}3.34, \mathrm{~d}, 11.5 ; 3.62, \mathrm{~d}, \\
11.5\end{array}$ & 72.9 & $\begin{array}{l}2.40, \mathrm{~d}, 13.5 ; 2.76, \mathrm{~d}, \\
13.5\end{array}$ & 35.2 \\
\hline $\mathrm{CH}_{2} \mathrm{OH}$ & $\begin{array}{l}3.51, \mathrm{dd}, 12.0,5.0 ; 3.55 \\
\mathrm{dd}, 12.0,5.0\end{array}$ & 62.9 & $\begin{array}{l}3.49, \mathrm{dd}, 11.5,5.0 ; 3.56 \\
\mathrm{dd}, 11.5,6.0\end{array}$ & 65.9 & $\begin{array}{l}3.48, \mathrm{dd}, 11.5,5.0 ; 3.56 \\
\mathrm{dd}, 11.5,5.0\end{array}$ & 65.9 \\
\hline $\mathrm{CH}_{2} \mathrm{OH}$ & $\begin{array}{l}3.51, \mathrm{dd}, 12.0,5.0 ; 3.55 \\
\mathrm{dd}, 12.0,5.0\end{array}$ & 62.9 & $\begin{array}{l}3.57, \mathrm{dd}, 12.0,5.0 ; 3.60 \\
\mathrm{dd}, 12.0,5.0\end{array}$ & 62.8 & $\begin{array}{l}3.48, \mathrm{dd}, 11.5,5.0 ; 3.56 \\
\mathrm{dd}, 11.5,5.0\end{array}$ & 65.9 \\
\hline
\end{tabular}

From all these data, and as the configuration of C-2 and C-8 was governed by that of starting materials 9a and $\mathbf{9 b}$, isomer 13a adopted necessarily a structure stabilized by a double anomeric effect and equatorial positions for its substituents, with a $(2 R, 6 S, 8 R)$ configuration. Starting from 1,3-dichloropropan-2-one, this spiranic oxathiane-dioxane system was obtained in four steps, and $28-34 \%$ range overall yield depending of the order of introduction of oxa- and thia-synthons $\mathbf{9 a}, \mathbf{b}$.

At this stage of the work, the examination of the more doubtful synthesis of aza-spiroketals remained unsolved. We began our investigation starting from the monochlorooxime 11c, from which oxa- and thiacompounds could be envisioned. Substitution of 11c by the thio-solketal $\mathbf{9 b}$ was efficiently accomplished using KH in THF and furnished 14 in $81 \%$ yield (Scheme 5). The assignment of all signals in the ${ }^{1} \mathrm{H}$ and ${ }^{13} \mathrm{C}$ NMR spectra was in this case complicated by the existence of rotamers due to the carbamate group, together with the presence of the two $Z$ and $E$ isomers.

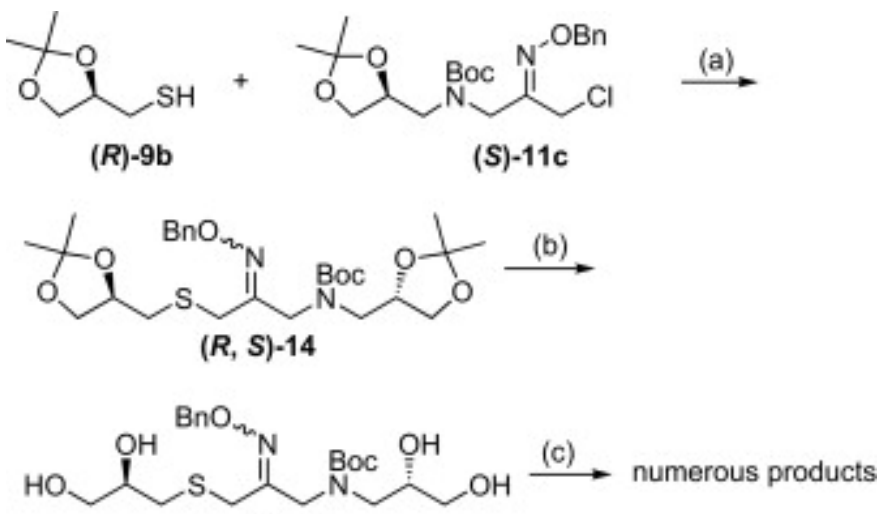

$(R, S)-15$

Scheme 5. Reagents and conditions: (a) KH, THF, 81\%; (b) Amberlyst ${ }^{\circledR} 15$, acetone $/ \mathrm{H}_{2} \mathrm{O}(10: 1), \Delta, 4 \mathrm{~h}$, $33 \%$; (c) $\Delta$.

Treatment of $\mathbf{1 4}$ in our classical acidic medium afforded after $4 \mathrm{~h}$ at reflux the tetraol $\mathbf{1 5}$ as the sole product of the reaction. Pursuing the heating, we observed the disappearance of 14 (TLC monitoring) accompanied by the formation of numerous side-products lacking the Boc group in their skeleton (checked by ${ }^{1} \mathrm{H}$ NMR spectrum). At ambient temperature and even after 4 days, the oxime function of 15 remained unchanged.

At this stage, the reactivity of the Boc group under our acid-heating conditions, together with the trouble to obtain in good yields aza-protected chlorooximes $\mathbf{1 1},{ }^{10}$ prompted us to investigate the synthesis of spiromorpholines from the monochlorooximes 11a,b, testing their reactivity toward amine 9c (Scheme 6). 


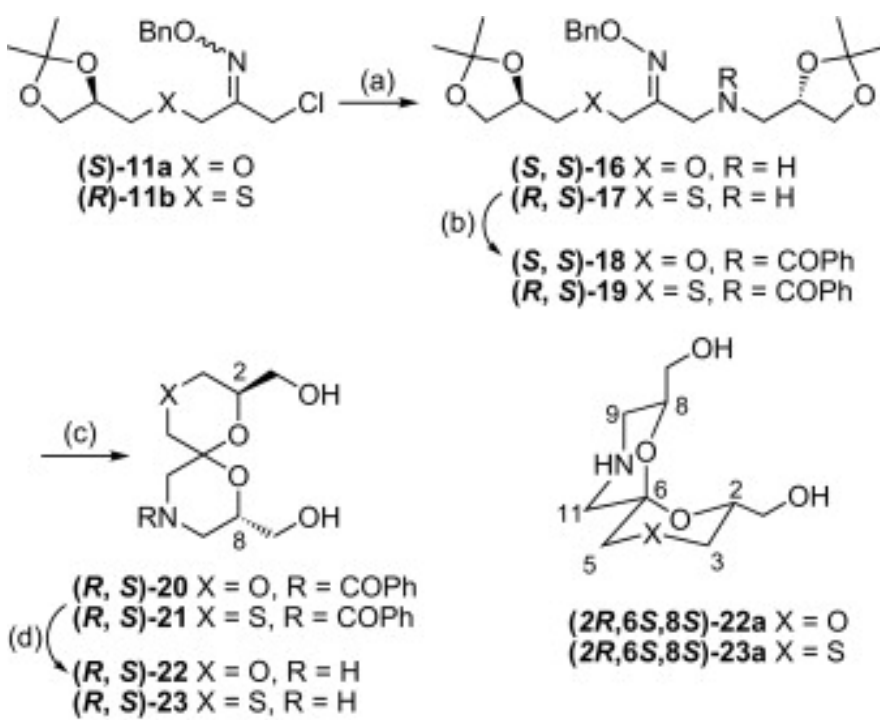

Scheme 6. Reagents and conditions: (a) $\mathrm{MeOH}, \Delta, 9 c, 8 \mathrm{~h}, 61 \%$ from 11a, 67\% from 11b; (b) $\mathrm{PhCOCl}$, $\mathrm{NEt}_{3}, \mathrm{DMAP}, \mathrm{CH}_{2} \mathrm{Cl}_{2}, 0{ }^{\circ} \mathrm{C}, 90 \%$ from 16, 95\% from 17; (c) Amberlyst ${ }^{\circledR} 15,\left(\mathrm{CH}_{2} \mathrm{O}\right)_{n}$, acetone $/ \mathrm{H}_{2} \mathrm{O}$ (10:1), $\Delta, 61 \%$ from 18, 65\% from 19; (d) $\mathrm{LiAlH}_{4}, \mathrm{THF}$, nearly quantitative.

Oximes 11a,b were heated in boiling methanol during $8 \mathrm{~h}$ in the presence of 3 equiv of amine 9c. Under these conditions, only monosubstitution was observed, leading to oxime $\mathbf{1 6}$ in $61 \%$ yield as a mixture of $E / Z$ isomers (5/3 ratio determined on the ${ }^{1} \mathrm{H}$ NMR spectrum) and oxime $\mathbf{1 7}$ in $67 \%$ yield as a $1 / 3$ mixture of $E / Z$ isomers. Compounds $\mathbf{1 6}$ and $\mathbf{1 7}$ were then treated by freshly distilled benzoylchloride in presence of $\mathrm{NEt}_{3}$ and DMAP in dichloromethane to furnish the attempted amides $\mathbf{1 8}$ and $\mathbf{1 9}$ in $90 \%$ and $95 \%$ yields, respectively. The final deprotection-spiroketalization step was then carried out as usual, through the addition of paraformaldehyde to regenerate the keto-function. The targeted spiromorpholinoketals 20 and 21 were obtained in $61 \%$ and $65 \%$ yields, respectively. No other derivatives were detected in the crude reaction mixture.

Finally, compounds 20 and 21 were obtained from 1,3-dichloropropan-2-one in five steps in 17\% and $26 \%$ overall yields, respectively. Full structural characterization of 20 and 21 could not be achieved at ambient temperature, as ${ }^{1} \mathrm{H}$ spectra were typical of coalescence phenomena and ${ }^{13} \mathrm{C}$ NMR spectra revealed mixtures of spiranic compounds. From ${ }^{13} \mathrm{C}$ NMR spectrum recorded at $70{ }^{\circ} \mathrm{C}$, each compound, 20 and 21, appeared clearly as a mixture of two inseparable isomers. The attribution of ${ }^{13} \mathrm{C}$ chemical shifts of the major and minor ones could be realized at this stage and, in the case of 20, spectroscopic data were correlated with those already published in the literature. ${ }^{8 \mathrm{c}}$

To overcome the problem of coalescence and determine without ambiguity the structure of each major isomer of $\mathbf{2 0}$ and 21, we reduced their amide function. The full cleavage of the amido group was

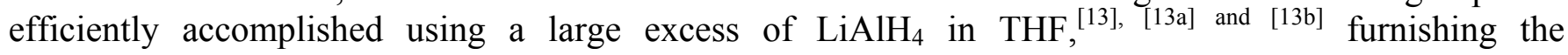
spiromorpholines 22 and 23 as, once again, a mixture of inseparable isomers in a 4/1 ratio for 22 and 9/1 ratio for 23. Finally, major compounds could be isolated and characterized through their derivatization as silylether derivatives (TBDPS-protection/ $\mathrm{Bu}_{4} \mathrm{NF}$ deprotection).

Spiranic carbons of major isomers 22a and 23a were detected at $91.6 \mathrm{ppm}$ (22a) and $90.9 \mathrm{ppm}$ (23a). These values were once again in agreement with those observed for $(6 S)$-spiro[5.5] undecane series. The C-3 and C-9 appeared at $67.2 \mathrm{ppm}$ (22a)-66.2 ppm (23a) and $46.1 \mathrm{ppm}$ (22a and 23a), respectively. Major isomers adopted a chair conformation for the two cycles as evidenced by (i) $\mathrm{H}-9_{\mathrm{ax}}$ and $\mathrm{H}-3_{\mathrm{ax}}$ that were detected as triplets with ${ }^{2} J={ }^{3} J=12.0 \mathrm{~Hz}$, and (ii) $\mathrm{H}-9_{\mathrm{eq}}$ and $\mathrm{H}-3_{\mathrm{eq}}$ resonating either as broad doublets $\left({ }^{2} J=12.0 \mathrm{~Hz}\right)$ or dedoubled doublet $\left({ }^{2} J=12.0 \mathrm{~Hz},{ }^{3} J=2.5 \mathrm{~Hz}\right)$. From all spectroscopic data, we assumed that majors isomers were the $(2 R, 6 S, 8 S)$-22a and the $(2 R, 6 S, 8 S)-23 \mathbf{a}$.

\section{Conclusion}

To summarize, we have developed an original and efficient pathway to new 1,7-dioxaspiro[5.5] undecanes incorporating in their skeleton two different heteroatoms in positions 4 and 10. Starting from commercially available $(S)$-solketal and 1,3-dichloropropan-2-one, we have shown that, in few steps and 
with good overall yields, we were able to elaborate in a modular way, spiranic systems based on the association of dioxane-oxathiane, dioxane-morpholine, and oxathiane-morpholine cores. Finally, this work completed and generalized our previous study in the 'symmetrical' series, namely spirobisdioxane and spirobisoxathiane systems.

\section{Experimental section}

\subsection{General experimental methods}

Infrared spectra were recorded on a Perkin-Elmer 881 instrument. ${ }^{1} \mathrm{H}$ NMR $(400 \mathrm{MHz})$ and ${ }^{13} \mathrm{C}$ NMR $(100 \mathrm{MHz})$ were recorded with a BRUKER AC 400 spectrometer. Chemical shifts ( $\delta$ values) are expressed in parts per million (ppm), coupling constants $(J)$ are expressed in hertz, and multiplicities are mentioned as follows: s (singlet), $d$ (doublet), $t$ (triplet), q (quartet), $Q$ (quintet), $m$ (multiplet). NMR spectra were recorded in $\mathrm{CDCl}_{3}, \mathrm{CD}_{3} \mathrm{OD}$ or DMSO- $d_{6}$, using the solvent signals as reference. Mass spectra were recorded with a Hewlett Packard 5989B instrument and high resolution mass spectra (HRMS) were performed with a Q-TOF micromass. Chromatography was performed using silica gel 60 (230-400 mesh) and thin layer chromatography (TLC) was performed on silica gel $60 \mathrm{PF}_{254}$ plates $(20 \times 20 \mathrm{~cm})$. Compounds were identified using UV fluorescence $(\lambda=254 \mathrm{~nm})$ and/or staining with a $5 \%$ phosphomolybdic acid solution in ethanol following by heating. Commercially reagents (Aldrich, Acros, Lancaster) were used as received without additional purification. Tetrahydrofuran (THF) was distilled from potassium/benzophenone while dichloromethane $\left(\mathrm{CH}_{2} \mathrm{Cl}_{2}\right)$ was dried over calcium hydride prior to use.

\subsection{Synthesis}

4.2.1. (2E)- and (2Z)-1-Chloro-3-\{[(4S)-2,2-dimethyl-1,3-dioxolan-4-yl]methoxy\}propanone $O$-benzyloxime (11a)

Method A. To a solution of oxime $8(2.11 \mathrm{~g}, 9.1 \mathrm{mmol})$ and $\mathrm{KH}(2.44 \mathrm{~g}, 18.2 \mathrm{mmol})$ in THF $(3 \mathrm{~mL})$ was added $(S)-9 \mathrm{a}(1.21 \mathrm{~g}, 9.1 \mathrm{mmol})$ and the resulting mixture was stirred until disubstituted compound was detected on TLC. The reaction was stopped by addition of a saturated solution of $\mathrm{NH}_{4} \mathrm{Cl}$. After extraction with ethyl acetate, the crude solution was concentrated in vacuo and purified by flash chromatography using $(19: 1 \rightarrow 7: 3)$ cyclohexane/ethyl acetate as eluent to give pure 11a as a colorless liquid $(0.90 \mathrm{~g}, 30 \%)$. Method B. To a solution of $(S)-9 a(0.330 \mathrm{~g}, 2.5 \mathrm{mmol})$ in toluene $(10 \mathrm{~mL})$ were added $\mathrm{KOH}(0.165 \mathrm{~g}$, $2.5 \mathrm{mmol})$ and 18 -crown-6 $(0.330 \mathrm{~g}, 1.25 \mathrm{mmol})$. The resulting mixture was heated at $70{ }^{\circ} \mathrm{C}$ for $1 \mathrm{~h}$ before addition, at $70{ }^{\circ} \mathrm{C}$ and over a period of $1 \mathrm{~h}$, of a solution of oxime $8(0.580 \mathrm{~g}, 2.5 \mathrm{mmol})$ in toluene $(5 \mathrm{~mL})$. At the appearance of disubstituted compound, reaction is stopped by addition of water. The resulting mixture is concentrated in vacuo and layers were separated. After extraction of the aqueous one by ethyl acetate, the combined organic layers are dried and concentrated. A final purification by flash chromatography using $(4: 1 \rightarrow 1: 1)$ cyclohexane/ethyl acetate as eluent furnished pure 11a in a $40-60 \%$ yield.

Compound 11a: IR $v_{\max }\left(\right.$ neat, $\left.\mathrm{cm}^{-1}\right) 1600(\mathrm{C}=\mathrm{N}), 1250-1040(\mathrm{C}-\mathrm{O}) ;{ }^{1} \mathrm{H}$ NMR $\left(\mathrm{CDCl}_{3}\right) \delta / \mathrm{ppm} 7.40-$ $7.30(\mathrm{~m}, 10 \mathrm{H}, \mathrm{H}-\mathrm{Ar}, E$ and $Z), 5.17\left(\mathrm{~s}, 2 \mathrm{H}, \mathrm{CH}_{2} \mathrm{Ph}, Z\right), 5.11\left(\mathrm{~s}, 2 \mathrm{H}, \mathrm{CH}_{2} \mathrm{Ph}, E\right), 4.48(\mathrm{~d}, J=15.0 \mathrm{~Hz}, 1 \mathrm{H}$, $\left.\mathrm{O}-\mathrm{CH}_{2}-\mathrm{C}=, E\right), 4.44\left(\mathrm{~d}, J=15.0 \mathrm{~Hz}, 1 \mathrm{H}, \mathrm{O}-\mathrm{CH}_{2}-\mathrm{C}=, E\right), 4.27(\mathrm{Q}, J=6.0 \mathrm{~Hz}, 1 \mathrm{H}, \mathrm{CH}-\mathrm{O}, E), 4.26(\mathrm{~m}$, $1 \mathrm{H}, \mathrm{CH}-\mathrm{O}, Z), 4.23\left(\mathrm{~d}, J=11.5 \mathrm{~Hz}, 1 \mathrm{H}, \mathrm{CH}_{2} \mathrm{Cl}, E\right), 4.21$ (d, J=11.5 Hz, 1H, $\left.\mathrm{CH}_{2} \mathrm{Cl}, E\right), 4.21(\mathrm{~m}, 2 \mathrm{H}, \mathrm{O}-$ $\left.\mathrm{CH}_{2}-\mathrm{C}=, Z\right), 4.20\left(\mathrm{~m}, 1 \mathrm{H}, \mathrm{CH}_{2} \mathrm{Cl}, Z\right), 4.19\left(\mathrm{~d}, J=11.5 \mathrm{~Hz}, 1 \mathrm{H}, \mathrm{CH}_{2} \mathrm{Cl}, Z\right), 4.05\left(\mathrm{t}, J=7.0 \mathrm{~Hz}, 1 \mathrm{H}, \mathrm{CH}_{2}-\mathrm{O}\right.$, $E), 4.03\left(\mathrm{t}, J=6.5 \mathrm{~Hz}, 1 \mathrm{H}, \mathrm{CH}_{2}-\mathrm{O}, Z\right), 3.77\left(\mathrm{dd}, J=8.0,6.5 \mathrm{~Hz}, 1 \mathrm{H}, \mathrm{CH}_{2}-\mathrm{O}, E\right), 3.70(\mathrm{dd}, J=8.0,6.5 \mathrm{~Hz}$, $\left.1 \mathrm{H}, \mathrm{CH}_{2}-\mathrm{O}, Z\right), 3.55\left(\mathrm{dd}, J=10.0,5.5 \mathrm{~Hz}, 1 \mathrm{H}, \mathrm{CHO}-\mathrm{CH}_{2} \mathrm{O}, E\right), 3.50(\mathrm{dd}, J=10.0,5.0 \mathrm{~Hz}, 1 \mathrm{H}, \mathrm{CHO}-$ $\left.C_{2} \mathrm{O}, E\right), 3.49$ (dd, $\left.J=10.0,5.5 \mathrm{~Hz}, 1 \mathrm{H}, \mathrm{CHO}-\mathrm{CH}_{2} \mathrm{O}, Z\right), 3.44\left(\mathrm{dd}, J=10.0,5.0 \mathrm{~Hz}, 1 \mathrm{H}, \mathrm{CHO}-C \mathrm{H}_{2} \mathrm{O}, Z\right.$ ), $1.42(\mathrm{~s}, 6 \mathrm{H}, \mathrm{Me}, E$ and $Z), 1.36(\mathrm{~s}, 6 \mathrm{H}, \mathrm{Me}, E$ and $Z) ;{ }^{13} \mathrm{C} \mathrm{NMR}\left(\mathrm{CDCl}_{3}\right) \delta / \mathrm{ppm} 154.9(\mathrm{C}=\mathrm{N}, E), 152.4$ $(\mathrm{C}=\mathrm{N}, Z), 137.1$ (C-Ar, Z), $137.0(\mathrm{C}-\mathrm{Ar}, E), 128.4(\mathrm{C}-\mathrm{Ar}, Z$ and $E), 128.1$ (C-Ar, Z), $128.0(\mathrm{C}-\mathrm{Ar}, E)$, $127.9(\mathrm{C}-\mathrm{Ar}, Z$ and $E), 109.5\left(\mathrm{C}-\left(\mathrm{CH}_{3}\right)_{2}, E\right), 109.4\left(\mathrm{C}-\left(\mathrm{CH}_{3}\right)_{2}, Z\right), 76.7\left(\mathrm{CH}_{2} \mathrm{Ph}, E\right), 76.5\left(\mathrm{CH}_{2} \mathrm{Ph}, Z\right)$, $74.4(\mathrm{CH}-\mathrm{O}, E), 74.3(\mathrm{CH}-\mathrm{O}, \mathrm{Z}), 72.3\left(\mathrm{CHO}-\mathrm{CH}_{2} \mathrm{O}, \mathrm{E}\right), 71.3\left(\mathrm{CHO}-\mathrm{CH}_{2} \mathrm{O}, \mathrm{Z}\right), 69.3\left(\mathrm{O}-\mathrm{CH}_{2}-\mathrm{C}=, Z\right)$, $66.5\left(\mathrm{CH}_{2}-\mathrm{O}, E\right.$ and $\left.Z\right), 63.9\left(\mathrm{O}-\mathrm{CH}_{2}-\mathrm{C}=, E\right), 41.1\left(\mathrm{CH}_{2} \mathrm{Cl}, E\right), 32.7\left(\mathrm{CH}_{2} \mathrm{Cl}, Z\right), 26.7\left(\mathrm{CH}_{3}, E\right), 26.6$ $\left(\mathrm{CH}_{3}, Z\right), 25.3\left(\mathrm{CH}_{3}, Z\right.$ and $\left.E\right)$. Anal. Calcd for $\mathrm{C}_{16} \mathrm{H}_{22} \mathrm{ClNO}_{4}$ : C, 58.62; $\mathrm{H}, 6.76 ; \mathrm{Cl}, 10.82 ; \mathrm{N}, 4.27$. Found C, 58.57; H, 6.79; Cl, 10.72; N, 4.25.

4.2.2. (2E)- and (2Z)-1-Chloro-3-(\{[(4R)-2,2-dimethyl-1,3-dioxolan-4-yl]methyl\}thio)propanone $O$-benzyloxime (11b) 
To a solution of oxime $8(0.254 \mathrm{~g}, 1.1 \mathrm{mmol})$ and $\mathrm{NaH}(0.090 \mathrm{~g}, 2.2 \mathrm{mmol})$ in THF $(3 \mathrm{~mL})$ was added $(R)-9 b(0.200 \mathrm{~g}, 1.1 \mathrm{mmol})$ and the resulting mixture was stirred until disubstituted compound was detected on TLC. The reaction was stopped by addition of a saturated solution of $\mathrm{NH}_{4} \mathrm{Cl}(2 \mathrm{~mL})$. After extraction with ethyl acetate, the crude solution was concentrated in vacuo and purified by flash chromatography using $(19: 1 \rightarrow 4: 1)$ cyclohexane/ethyl acetate as eluent to give pure 11b as a colorless oil $(0.255 \mathrm{~g}, 64 \%)$. IR $v_{\max }\left(\right.$ neat, $\left.\mathrm{cm}^{-1}\right) 1618(\mathrm{C}=\mathrm{N}), 1250-1040(\mathrm{C}-\mathrm{O}) ;{ }^{1} \mathrm{H}$ NMR $\left(\mathrm{CDCl}_{3}\right) \delta / \mathrm{ppm} 7.38-$ $7.30(\mathrm{~m}, 10 \mathrm{H}, \mathrm{H}-\mathrm{Ar}, E$ and $Z), 5.14\left(\mathrm{~s}, 2 \mathrm{H}, \mathrm{CH}_{2} \mathrm{Ph}, Z\right), 5.11\left(\mathrm{~s}, 2 \mathrm{H}, \mathrm{CH}_{2} \mathrm{Ph}, E\right), 4.37\left(\mathrm{~s}, 2 \mathrm{H}, \mathrm{CH}_{2} \mathrm{Cl}, Z\right)$, 4.29 (d, $\left.J=11.5 \mathrm{~Hz}, 1 \mathrm{H}, \mathrm{CH}_{2} \mathrm{Cl}, E\right), 4.26\left(\mathrm{~d}, J=11.5 \mathrm{~Hz}, 1 \mathrm{H}, \mathrm{CH}_{2} \mathrm{Cl}, E\right), 4.19$ (Q, J=6.0 Hz, 1H, CH-O,E), $4.17(\mathrm{Q}, J=6.0 \mathrm{~Hz}, 1 \mathrm{H}, \mathrm{CH}-\mathrm{O}, Z), 3.99$ (dd, $J=8.5,6.0 \mathrm{~Hz}, 1 \mathrm{H}, \mathrm{CH}_{2}-\mathrm{O}, Z$ ), 3.95 (dd, $J=8.5,6.0 \mathrm{~Hz}, 1 \mathrm{H}$, $\left.\mathrm{CH}_{2}-\mathrm{O}, E\right), 3.60\left(\mathrm{~d}, J=13.5 \mathrm{~Hz}, 1 \mathrm{H}, \mathrm{S}-\mathrm{CH}_{2}-\mathrm{C}=, E\right), 3.55\left(\mathrm{~d}, J=13.5 \mathrm{~Hz}, 1 \mathrm{H}, \mathrm{S}-\mathrm{CH}_{2}-\mathrm{C}=, E\right), 3.59(\mathrm{dd}$, $\left.J=8.5, J=6.0 \mathrm{~Hz}, 1 \mathrm{H}, \mathrm{CH}_{2}-\mathrm{O}, E\right), 3.57\left(\mathrm{dd}, J=8.5,6.0 \mathrm{~Hz}, 1 \mathrm{H}, \mathrm{CH}_{2}-\mathrm{O}, Z\right), 3.40$ (d, $J=14.0 \mathrm{~Hz}, 1 \mathrm{H}, \mathrm{S}-$ $\left.\mathrm{CH}_{2}-\mathrm{C}=, Z\right), 3.37\left(\mathrm{~d}, J=14.0 \mathrm{~Hz}, 1 \mathrm{H}, \mathrm{S}-\mathrm{CH}_{2}-\mathrm{C}=, Z\right), 2.63\left(\mathrm{dd}, J=13.5,6.0 \mathrm{~Hz}, 1 \mathrm{H}, \mathrm{CH}-\mathrm{CH} \mathrm{S}_{2} \mathrm{~S}, E\right), 2.56$ $\left(\mathrm{dd}, J=13.5,6.5 \mathrm{~Hz}, 1 \mathrm{H}, \mathrm{CH}-\mathrm{CH}_{2} \mathrm{~S}, Z\right), 2.52\left(\mathrm{dd}, J=13.5,6.5 \mathrm{~Hz}, 1 \mathrm{H}, \mathrm{CH}-\mathrm{CH}_{2} \mathrm{~S}, E\right), 2.45$ (dd, $J=13.5$, $\left.6.0 \mathrm{~Hz}, 1 \mathrm{H}, \mathrm{CH}-\mathrm{CH}_{2} \mathrm{~S}, Z\right), 1.41$ (s, 3H, Me, Z), 1.40 (s, 3H, Me, E), 1.34 (s, 3H, Me, Z), 1.32 (s, 3H, Me, $E) ;{ }^{13} \mathrm{C} \mathrm{NMR}\left(\mathrm{CDCl}_{3}\right) \delta / \mathrm{ppm} 153.4(\mathrm{C}=\mathrm{N}, E), 152.0(\mathrm{C}=\mathrm{N}, Z), 137.3(\mathrm{C}-\mathrm{Ar}, Z), 136.7(\mathrm{C}-\mathrm{Ar}, E)$, $128.4(\mathrm{C}-\mathrm{Ar}, Z$ and $E), 128.1(\mathrm{C}-\mathrm{Ar}, E), 128.0(\mathrm{C}-\mathrm{Ar}, Z), 127.9(\mathrm{C}-\mathrm{Ar}, Z$ and $E), 109.5\left(\mathrm{C}-\left(\mathrm{CH}_{3}\right)_{2}, Z\right.$ and $E), 76.6\left(\mathrm{CH}_{2} \mathrm{Ph}, E\right), 76.4\left(\mathrm{CH}_{2} \mathrm{Ph}, Z\right), 74.9(\mathrm{CH}-\mathrm{O}, Z), 74.8(\mathrm{CH}-\mathrm{O}, E), 68.6\left(\mathrm{CH}_{2}-\mathrm{O}, Z\right), 68.5\left(\mathrm{CH}_{2}-\mathrm{O}\right.$, $E), 42.5\left(\mathrm{CH}_{2} \mathrm{Cl}, E\right), 35.1\left(\mathrm{CHO}-\mathrm{CH}_{2} \mathrm{~S}, E\right), 34.0\left(\mathrm{CHO}-\mathrm{CH}_{2} \mathrm{~S}, \mathrm{Z}\right), 33.7\left(\mathrm{CH}_{2} \mathrm{Cl}, \mathrm{Z}\right), 32.9\left(\mathrm{~S}-\mathrm{CH}_{2}-\mathrm{C}=\right.$, $Z), 26.9\left(\mathrm{CH}_{3}, Z\right.$ and $\left.E\right), 25.5\left(\mathrm{CH}_{3}, Z\right), 25.4\left(\mathrm{CH}_{3}, E\right), 24.4\left(\mathrm{~S}-\mathrm{CH}_{2}-\mathrm{C}=, E\right)$; HRMS (ESI): calculated for $\mathrm{C}_{16} \mathrm{H}_{22} \mathrm{ClNO}_{3} \mathrm{Na}[\mathrm{M}+\mathrm{Na}]^{+}$366.0907, found 366.0892.

4.2.3. (2Z)- and (2E)-1-\{[(4S)-2,2-Dimethyl-1,3-dioxolan-4-yl]methoxy\}-3-(\{[(4R)-2,2-dimethyl-1,3-dioxolan-4yl]methyl\}thio)propanone $O$-benzyloxime (12)

To a pre-washed KH (1.35 g, $10.4 \mathrm{mmol})$ solution in THF $(10 \mathrm{~mL})$ was added, under argon, thiol $(R)-9 \mathbf{b}$ $(0.850 \mathrm{mg}, 5.7 \mathrm{mmol})$ in THF $(10 \mathrm{~mL})$ followed after $15 \mathrm{~min}$ by a solution of 11a $(1.70 \mathrm{~g}, 5.2 \mathrm{mmol})$ in THF $(20 \mathrm{~mL})$. After the completion of the reaction (disappearance of 11a), water was added and the resulting solution was extracted with dichloromethane. The organic layer was concentrated in vacuo and purified by flash chromatography using (9:1) cyclohexane/ethyl acetate to give $12(2.0 \mathrm{~g}, 86 \%)$ as a colorless oil of a mixture of $Z$ and $E$ isomers.

The same protocol applied to $\mathbf{1 1 b}(0.200 \mathrm{~g}, 0.58 \mathrm{mmol})$ and $(S)$-solketal 9a $(0.092 \mathrm{mg}, 0.70 \mathrm{mmol})$ using $\mathrm{KH}(0.155 \mathrm{~g}, 1.16 \mathrm{mmol})$ gave, after purification, $12(0.138 \mathrm{~g}, 54 \%)$.

Compound 12: IR $v_{\max }\left(\right.$ neat, $\left.\mathrm{cm}^{-1}\right) 1600(\mathrm{C}=\mathrm{N}), 1250-1050(\mathrm{C}-\mathrm{O}) ;{ }^{1} \mathrm{H}$ NMR $\left(\mathrm{CDCl}_{3}\right) \delta / \mathrm{ppm} 7.40-7.25$ $(\mathrm{m}, 10 \mathrm{H}, \mathrm{H}-\mathrm{Ar}, E$ and $Z), 5.09$ (s, 2H, $\left.\mathrm{CH}_{2} \mathrm{Ph}, Z\right), 5.06$ (s, 2H, $\left.\mathrm{CH}_{2} \mathrm{Ph}, E\right), 4.46(\mathrm{~d}, J=15.0 \mathrm{~Hz}, 1 \mathrm{H}, \mathrm{O}-$ $\left.\mathrm{CH}_{2}-\mathrm{C}=\mathrm{N}, E\right), 4.43\left(\mathrm{~d}, J=15.0 \mathrm{~Hz}, 1 \mathrm{H}, \mathrm{O}-\mathrm{CH}_{2}-\mathrm{C}=\mathrm{N}, E\right), 4.25(\mathrm{Q}, J=6.0 \mathrm{~Hz}, 1 \mathrm{H}, \mathrm{CH}-\mathrm{O}, E), 4.24(\mathrm{Q}$, $J=6.0 \mathrm{~Hz}, 1 \mathrm{H}, \mathrm{CH}-\mathrm{O}, Z), 4.20(\mathrm{Q}, J=6.5 \mathrm{~Hz}, 1 \mathrm{H}, \mathrm{CH}-\mathrm{O}, Z), 4.20\left(\mathrm{~d}, J=12.0 \mathrm{~Hz}, 1 \mathrm{H}, \mathrm{O}-\mathrm{CH}_{2}-\mathrm{C}=\mathrm{N}, Z\right)$, $4.18(\mathrm{Q}, J=6.0 \mathrm{~Hz}, 1 \mathrm{H}, \mathrm{CH}-\mathrm{O}, E), 4.17\left(\mathrm{~d}, J=12.0 \mathrm{~Hz}, 1 \mathrm{H}, \mathrm{O}-\mathrm{CH}_{2}-\mathrm{C}=\mathrm{N}, Z\right), 4.03(\mathrm{dd}, J=8.5$ and $\left.6.5 \mathrm{~Hz}, 1 \mathrm{H}, \mathrm{O}-\mathrm{CH}_{2}-\mathrm{CHO}, E\right), 4.02\left(\mathrm{dd}, J=8.5\right.$ and $\left.6.5 \mathrm{~Hz}, 1 \mathrm{H}, \mathrm{O}-\mathrm{CH}_{2}-\mathrm{CHO}, Z\right), 4.00(\mathrm{dd}, J=8.5$ and $\left.6.0 \mathrm{~Hz}, 1 \mathrm{H}, \mathrm{O}-\mathrm{CH}_{2}-\mathrm{CHO}, E\right), 3.95\left(\mathrm{dd}, J=8.5\right.$ and $\left.6.0 \mathrm{~Hz}, 1 \mathrm{H}, \mathrm{O}-\mathrm{CH}_{2}-\mathrm{CHO}, Z\right), 3.74(\mathrm{dd}, J=8.5$ and $\left.6.5 \mathrm{~Hz}, 1 \mathrm{H}, \mathrm{O}-\mathrm{CH}_{2}-\mathrm{CHO}, E\right), 3.68\left(\mathrm{dd}, J=8.5\right.$ and $\left.6.5 \mathrm{~Hz}, 1 \mathrm{H}, \mathrm{O}-\mathrm{CH}_{2}-\mathrm{CHO}, Z\right), 3.59(\mathrm{dd}, J=8.5$ and $6.5 \mathrm{~Hz}, 2 \mathrm{H}, \mathrm{O}-\mathrm{CH}_{2}-\mathrm{CHO}, E$ and $\left.Z\right), 3.53\left(\mathrm{dd}, J=10.0\right.$ and $\left.5.5 \mathrm{~Hz}, 1 \mathrm{H}, \mathrm{CHO}-\mathrm{CH}_{2}-\mathrm{O}-\mathrm{CH}_{2}-, E\right), 3.48(\mathrm{~d}$, $\left.J=13.5 \mathrm{~Hz}, 1 \mathrm{H}, \mathrm{S}-\mathrm{CH}_{2}-\mathrm{C}=\mathrm{N}, Z\right), 3.48\left(\mathrm{dd}, J=10.0\right.$ and $\left.5.5 \mathrm{~Hz}, 1 \mathrm{H}, \mathrm{CH}_{2}-\mathrm{OCH}_{2} \mathrm{C}=\mathrm{N}, E\right), 3.47(\mathrm{dd}$, $J=10.0$ and $\left.6.0 \mathrm{~Hz}, 1 \mathrm{H}, \mathrm{CH}_{2}-\mathrm{OCH}_{2} \mathrm{C}=\mathrm{N}, Z\right), 3.46\left(\mathrm{~d}, J=13.5 \mathrm{~Hz}, 1 \mathrm{H}, \mathrm{S}-\mathrm{CH}_{2}-\mathrm{C}=\mathrm{N}, Z\right), 3.42(\mathrm{dd}$, $J=10.0$ and $\left.5.0 \mathrm{~Hz}, 1 \mathrm{H}, \mathrm{CH}_{2}-\mathrm{OCH}_{2} \mathrm{C}=\mathrm{N}, Z\right), 3.34\left(\mathrm{~d}, J=13.5 \mathrm{~Hz}, 1 \mathrm{H}, \mathrm{S}-\mathrm{CH}_{2}-\mathrm{C}=\mathrm{N}, E\right), 3.30(\mathrm{~d}$, $\left.J=13.5 \mathrm{~Hz}, 1 \mathrm{H}, \mathrm{S}-\mathrm{CH}_{2}-\mathrm{C}=\mathrm{N}, E\right), 2.68\left(\mathrm{dd}, J=13.5\right.$ and $\left.6.0 \mathrm{~Hz}, 1 \mathrm{H}, \mathrm{S}-\mathrm{CH}_{2}-\mathrm{CHO}, Z\right), 2.62(\mathrm{dd}, J=13.5$ and $\left.6.0 \mathrm{~Hz}, 1 \mathrm{H}, \mathrm{S}-\mathrm{CH}_{2}-\mathrm{CHO}, E\right), 2.55\left(\mathrm{dd}, J=13.5\right.$ and $\left.6.5 \mathrm{~Hz}, 1 \mathrm{H}, \mathrm{S}-\mathrm{CH}_{2}-\mathrm{CHO}, Z\right), 2.49$ (dd, $J=13.5$ and $\left.6.5 \mathrm{~Hz}, 1 \mathrm{H}, \mathrm{S}-\mathrm{CH}_{2}-\mathrm{CHO}, E\right), 1.41(\mathrm{~s}, 9 \mathrm{H}, \mathrm{Me}), 1.40(\mathrm{~s}, 3 \mathrm{H}, \mathrm{Me}), 1.35(\mathrm{~s}, 6 \mathrm{H}, \mathrm{Me}), 1.34(\mathrm{~s}, 3 \mathrm{H}, \mathrm{Me})$, $1.32(\mathrm{~s}, 3 \mathrm{H}, \mathrm{Me}) ;{ }^{13} \mathrm{C} \mathrm{NMR}\left(\mathrm{CDCl}_{3}\right) \delta / \mathrm{ppm} 155.9(\mathrm{C}=\mathrm{N}, E), 154.6(\mathrm{C}=\mathrm{N}, Z), 137.6(\mathrm{C}-\mathrm{Ar}, E), 137.1$ (C-Ar, Z), 128.4 (C-Ar, Z and E), 128.3 (C-Ar, Z), 128.2 (C-Ar, Z), 128.0 (C-Ar, E), 127.9 (C-Ar, E), $109.5\left(C-\left(\mathrm{CH}_{3}\right)_{2}, Z\right), 109.4\left(C-\left(\mathrm{CH}_{3}\right)_{2}, E\right), 76.2\left(\mathrm{CH}_{2} \mathrm{Ph} \mathrm{Z}\right), 76.1\left(\mathrm{CH}_{2} \mathrm{Ph}, E\right), 74.9(\mathrm{CH}-\mathrm{O}, E), 74.8(\mathrm{CH}-$ $\mathrm{O}, \mathrm{Z}), 74.5(\mathrm{CH}-\mathrm{O}, \mathrm{Z}), 74.4(\mathrm{CH}-\mathrm{O}, E), 72.2\left(\mathrm{CHO}-\mathrm{CH}_{2} \mathrm{O}, E\right), 71.2\left(\mathrm{CHO}-\mathrm{CH}_{2} \mathrm{O}, \mathrm{Z}\right), 69.4\left(\mathrm{O}-\mathrm{CH}_{2}-\right.$ $\mathrm{C}=\mathrm{N}, Z), 68.8\left(\mathrm{O}-\mathrm{CH}_{2}-\mathrm{CHO}, E\right), 68.6\left(\mathrm{O}-\mathrm{CH}_{2}-\mathrm{CHO}, Z\right), 66.5\left(\mathrm{O}-\mathrm{CH}_{2}-\mathrm{CHO}, \mathrm{Z}\right.$ and $\left.E\right), 64.5\left(\mathrm{O}-C_{2}-\right.$ $\mathrm{C}=\mathrm{N}, E), 35.2\left(\mathrm{~S}-\mathrm{CH}_{2}-\mathrm{CHO}, Z\right), 34.1\left(\mathrm{~S}-\mathrm{CH}_{2}-\mathrm{CHO}, E\right), 31.2\left(\mathrm{~N}=\mathrm{C}-\mathrm{CH}_{2}-\mathrm{S}, E\right), 26.9\left(\mathrm{CH}_{3}, Z\right.$ and $\left.E\right)$, $26.7\left(\mathrm{CH}_{3}, Z\right.$ and $\left.E\right), 25.6\left(\mathrm{CH}_{3}, Z\right.$ and $\left.E\right), 25.4\left(\mathrm{CH}_{3}, E\right), 25.3\left(\mathrm{CH}_{3}, Z\right), 24.5\left(\mathrm{~N}=\mathrm{C}-\mathrm{CH}_{2}-\mathrm{S}, Z\right)$. Anal. Calcd for $\mathrm{C}_{22} \mathrm{H}_{33} \mathrm{NO}_{6} \mathrm{~S}$ : C, 60.11; H, 7.57; N, 3.1. Found: C, 60.21; H, 7.51; N, 3.29.

4.2.4. 2,8-Dihydroxymethyl-4-thia-1,7,10-trioxaspiro-[5.5]undecane (13) 
A solution of $(S, R)-12(0.500 \mathrm{~g}, 1.14 \mathrm{mmol})$ in $10 \mathrm{~mL}$ of a mixture $(10: 1, \mathrm{v} / \mathrm{v})$ of acetone/water, Amberlyst ${ }^{\circledR} 15(0.250 \mathrm{~g})$, and paraformaldehyde $(0.034 \mathrm{~g}, 1.14 \mathrm{mmol})$ was heated under reflux for $48 \mathrm{~h}$. After cooling, the mixture was filtered on a Celite ${ }^{\circledR}$ pad. The residue was purified by column chromatography on silica gel using gradient $(1: 0 \rightarrow 49: 1)$ ethyl acetate/methanol as eluent, which gave a 19:1 mixture of isomers $13(0.185 \mathrm{~g}, 79 \%)$. IR $v_{\max }\left(\right.$ neat, $\left.\mathrm{cm}^{-1}\right) 3400(\mathrm{O}-\mathrm{H}) ;{ }^{13} \mathrm{C} \mathrm{NMR}\left(\mathrm{CD}_{3} \mathrm{OD}\right) \delta / \mathrm{ppm}$ 107.0-92.3 (C-6), 75.6-72.9 (C-11), 75.9-72.0 (C-2), 72.1-70.4 (C-8), 74.4-68.7 (C-9), 65.9 (C-12), 64.3-62.8 (C-13), 32.2-31.0 (C-5), 29.8-27.5 (C-3).

Major compound: $(2 R, 6 S, 8 R)-13 a:{ }^{1} \mathrm{H}$ NMR $\left(\mathrm{CDCl}_{3}\right) \delta / \mathrm{ppm} 4.06\left(\mathrm{dddd}, J_{2 \mathrm{ax} 3 \mathrm{ax}}=11.0 \mathrm{~Hz}, J_{2 \mathrm{ax} 12 \mathrm{a}}=6.0 \mathrm{~Hz}\right.$, $\left.J_{2 \mathrm{ax} 12 \mathrm{~b}}=5.0 \mathrm{~Hz}, J_{2 \mathrm{ax} 3 \mathrm{eq}}=2.0 \mathrm{~Hz}, 1 \mathrm{H}, \mathrm{H}-2_{\mathrm{ax}}\right), 4.02\left(\mathrm{dtd}, J_{8 \mathrm{ax} 9 \mathrm{ax}}=11.0 \mathrm{~Hz}, J_{8 \mathrm{ax} 13}=5.0 \mathrm{~Hz}, J_{8 \mathrm{ax} 9 \mathrm{eq}}=2.5 \mathrm{~Hz}, 1 \mathrm{H}, \mathrm{H}-\right.$ $\left.8_{\mathrm{ax}}\right), 3.84\left(\mathrm{dd}, J_{9 \mathrm{eq} 9 \mathrm{ax}}=11.5 \mathrm{~Hz}, J_{9 \mathrm{eq} 8 \mathrm{ax}}=2.5 \mathrm{~Hz}, 1 \mathrm{H}, \mathrm{H}-9_{\mathrm{eq}}\right), 3.62\left(\mathrm{~d}, J_{11 \mathrm{eq} 1 \mathrm{lax}}=11.5 \mathrm{~Hz}, 1 \mathrm{H}, \mathrm{H}-11_{\mathrm{eq}}\right), 3.60$ $\left(\mathrm{dd}, J_{13 \mathrm{a} 13 \mathrm{~b}}=12.0 \mathrm{~Hz}, J_{13 \mathrm{a} 8}=5.0 \mathrm{~Hz}, 1 \mathrm{H}, \mathrm{H}-13 \mathrm{a}\right), 3.57\left(\mathrm{dd}, J_{13 \mathrm{~b} 13 \mathrm{a}}=12.0 \mathrm{~Hz}, J_{13 \mathrm{~b} 8}=6.0 \mathrm{~Hz}, 1 \mathrm{H}, \mathrm{H}-13 \mathrm{~b}\right), 3.56$ $\left(\mathrm{dd}, J_{12 \mathrm{a} 12 \mathrm{~b}}=11.5 \mathrm{~Hz}, J_{12 \mathrm{a} 2}=6.0 \mathrm{~Hz}, 1 \mathrm{H}, \mathrm{H}-12 \mathrm{a}\right), 3.49\left(\mathrm{dd}, J_{12 \mathrm{~b} 12 \mathrm{a}}=11.5 \mathrm{~Hz}, J_{12 \mathrm{~b} 2}=5.0 \mathrm{~Hz}, 1 \mathrm{H}, \mathrm{H}-12 \mathrm{~b}\right), 3.41$ $\left(\mathrm{t}, J_{9 \mathrm{ax} 9 \mathrm{eq}}=J_{9 \mathrm{ax} 8 \mathrm{ax}}=11.0 \mathrm{~Hz}, 1 \mathrm{H}, \mathrm{H}-9_{\mathrm{ax}}\right), 3.34\left(\mathrm{~d}, J_{11 \mathrm{ax} 11 \mathrm{eq}}=11.5 \mathrm{~Hz}, 1 \mathrm{H}, \mathrm{H}-11_{\mathrm{ax}}\right), 2.68\left(\mathrm{~d}, J_{5 \mathrm{ax} 5 \mathrm{eq}}=13.5 \mathrm{~Hz}\right.$, $\left.1 \mathrm{H}, \mathrm{H}-5_{\mathrm{ax}}\right), 2.57$ (dd, $\left.J_{3 \mathrm{ax} 3 \mathrm{eq}}=13.0 \mathrm{~Hz}, J_{3 \mathrm{ax} 2 \mathrm{ax}}=11.0 \mathrm{~Hz}, 1 \mathrm{H}, \mathrm{H}-3_{\mathrm{ax}}\right), 2.37$ (dt, $J_{3 \mathrm{eq} 3 \mathrm{ax}}=13.0 \mathrm{~Hz}$, $\left.J_{3 \mathrm{eq} 2 \mathrm{ax}}=J_{3 \mathrm{eq} 5 \mathrm{eq}}=2.0 \mathrm{~Hz}, 1 \mathrm{H}, \mathrm{H}-3_{\mathrm{eq}}\right), 2.31\left(\mathrm{dd}, J_{5 \mathrm{eq} 5 \mathrm{ax}}=13.5 \mathrm{~Hz}, J_{5 \mathrm{eq} 3 \mathrm{eq}}=1.5 \mathrm{~Hz}, 1 \mathrm{H}, \mathrm{H}-5_{\mathrm{eq}}\right) ;{ }^{13} \mathrm{C} \mathrm{NMR}\left(\mathrm{CDCl}_{3}\right)$ $\delta /$ ppm 92.3 (C-6), 72.9 (C-11), 72.0 (C-2), 70.4 (C-8), 68.7 (C-9), 65.9 (C-12), 62.8 (C-13), 31.0 (C-5), 27.5 (C-3); HRMS (ESI): calculated for $\mathrm{C}_{9} \mathrm{H}_{16} \mathrm{O}_{5} \mathrm{NaS}[\mathrm{M}+\mathrm{Na}]^{+}$259.0616, found 259.0620.

4.2.5. tert-Butyl $\{[(4 S)$-2,2-dimethyl-1,3-dioxolan-4-yl]methyl\}(3-\{[(4R)-2,2-dimethyl-1,3-dioxolan-4-yl]methyl\}thio)-\{2[(benzyloxy)imino]propyl\}carbamate (14)

To a suspension of KH $(0.125 \mathrm{~g}, 0.92 \mathrm{mmol})$ in mineral oil, was added, under argon, a solution of $(S)$-11c $(0.200 \mathrm{~g}, 0.46 \mathrm{mmol})$ in THF $(3 \mathrm{~mL})$, followed, after $30 \mathrm{~min}$, by a solution of thiol $(R)-9 \mathbf{b}(0.082 \mathrm{mg}$, $0.55 \mathrm{mmol})$ in THF $(1 \mathrm{~mL})$. The reaction was monitored by TLC. After adjunction of water, the mixture was extracted with dichloromethane $(3 \times 10 \mathrm{~mL})$ and the organic extracts combined before being dried $\left(\mathrm{MgSO}_{4}\right)$, filtered, and then the solvent was removed using a rotary evaporator. The residue was purified by flash chromatography on silica gel using $(1: 0 \rightarrow 4: 1)$ cyclohexane/ethyl acetate as an eluent to give 14 as a mixture of two rotamers $\left(0.200 \mathrm{~g}, 81 \%\right.$, colorless oil). IR $v_{\max }$ (neat, $\left.\mathrm{cm}^{-1}\right) 1697(\mathrm{C}=\mathrm{O}), 1250-1060$ $(\mathrm{C}-\mathrm{O}) ;{ }^{1} \mathrm{H}$ NMR $\left(\mathrm{CDCl}_{3}\right) \delta / \mathrm{ppm} 7.36-7.27$ (m, 10H, H-Ar), $5.08\left(\mathrm{~s}, 2 \mathrm{H}, \mathrm{CH}_{2} \mathrm{Ph}\right), 5.06\left(\mathrm{~s}, 2 \mathrm{H}, \mathrm{CH}_{2} \mathrm{Ph}\right)$, 4.41-4.28 (m, 4H, CH $\left.\mathrm{CH}_{2} \mathrm{~S}\right), 4.21\left(\mathrm{~m}, 2 \mathrm{H}, \mathrm{CHO}-\mathrm{CH}_{2} \mathrm{~N}\right), 4.17\left(\mathrm{Q}, J=6.5 \mathrm{~Hz}, 2 \mathrm{H}, \mathrm{SCH}_{2}-\mathrm{CHO}\right), 3.98$ (dd, $\left.{ }^{2} J=8.5 \mathrm{~Hz},{ }^{3} J=6.0 \mathrm{~Hz}, 2 \mathrm{H}, \mathrm{CH}_{2} \mathrm{O}\right), 4.02-3.95\left(\mathrm{~m}, 2 \mathrm{H}, \mathrm{CH}_{2} \mathrm{O}\right), 3.59\left(\mathrm{dd},{ }^{2} J=8.5 \mathrm{~Hz},{ }^{3} J=6.5 \mathrm{~Hz}, 2 \mathrm{H}, \mathrm{CH}_{2} \mathrm{O}\right)$, $3.56\left(\mathrm{dd},{ }^{2} \mathrm{~J}=8.5 \mathrm{~Hz},{ }^{3} \mathrm{~J}=6.5 \mathrm{~Hz}, 2 \mathrm{H}, \mathrm{CH}_{2} \mathrm{O}\right), 3.45-3.25\left(\mathrm{~m}, 4 \mathrm{H}, \mathrm{O}-\mathrm{CH}-\mathrm{CH}_{2} \mathrm{~N}\right), 3.22\left(\mathrm{~s}, 2 \mathrm{H}, \mathrm{NCH}_{2}\right), 3.18$ $\left(\mathrm{s}, 2 \mathrm{H}, \mathrm{NCH}_{2}\right), 2.58\left(\mathrm{dd},{ }^{2} J=13.5 \mathrm{~Hz},{ }^{3} J=6.0 \mathrm{~Hz}, 2 \mathrm{H}, \mathrm{SCH}_{2}\right), 2.46\left(\mathrm{dd},{ }^{2} J=13.5 \mathrm{~Hz},{ }^{3} J=6.5 \mathrm{~Hz}, 2 \mathrm{H}, \mathrm{SCH}_{2}\right)$, $1.44(\mathrm{~s}, 9 \mathrm{H}, t-\mathrm{Bu}), 1.42(\mathrm{~s}, 6 \mathrm{H}, \mathrm{Me}), 1.41(\mathrm{~s}, 6 \mathrm{H}, \mathrm{Me}), 1.39(\mathrm{~s}, 9 \mathrm{H}, t-\mathrm{Bu}), 1.34(\mathrm{~s}, 6 \mathrm{H}, \mathrm{Me}), 1.33(\mathrm{~s}, 6 \mathrm{H}$, $\mathrm{Me}) ;{ }^{13} \mathrm{C}$ NMR $\left(\mathrm{CDCl}_{3}\right) \delta / \mathrm{ppm} 155.5\left(\mathrm{CO}_{2} t-\mathrm{Bu}\right), 155.4\left(\mathrm{CO}_{2} t-\mathrm{Bu}\right), 155.0(\mathrm{C}=\mathrm{N}), 137.7(\mathrm{C}-\mathrm{Ar}), 128.3$ (C-Ar), $128.1(\mathrm{C}-\mathrm{Ar}), 127.8(\mathrm{C}-\mathrm{Ar}), 109.5\left(\mathrm{C}-\left(\mathrm{CH}_{3}\right)_{2}\right), 109.3\left(\mathrm{C}-\left(\mathrm{CH}_{3}\right)_{2}\right), 80.7\left(\mathrm{C}-\left(\mathrm{CH}_{3}\right)_{3}\right), 80.5(\mathrm{C}-$ $\left.\left(\mathrm{CH}_{3}\right)_{3}\right), 76.2\left(\mathrm{CH}_{2} \mathrm{Ph}\right), 76.1\left(\mathrm{CH}_{2} \mathrm{Ph}\right), 75.1(\mathrm{CHO}), 74.9(\mathrm{CHO}), 68.7\left(\mathrm{CH}_{2} \mathrm{O}\right), 67.2\left(\mathrm{CH}_{2} \mathrm{O}\right), 67.1$ $\left(\mathrm{CH}_{2} \mathrm{O}\right), 51.0\left(\mathrm{CH}_{2} \mathrm{~N}\right), 50.7\left(\mathrm{CH}_{2} \mathrm{~N}\right), 44.1\left(\mathrm{NCH}_{2}\right), 44.0\left(\mathrm{NCH}_{2}\right), 34.0\left(\mathrm{SCH}_{2}\right), 33.8\left(\mathrm{SCH}_{2}\right), 32.6\left(\mathrm{CH}_{2} \mathrm{~S}\right)$, $32.0\left(\mathrm{CH}_{2} \mathrm{~S}\right), 28.3(t-\mathrm{Bu}), 26.9\left(\mathrm{CH}_{3}\right), 26.8\left(\mathrm{CH}_{3}\right), 25.6\left(\mathrm{CH}_{3}\right), 25.5\left(\mathrm{CH}_{3}\right) ; \mathrm{HRMS}(\mathrm{ESI})$ : calculated for $\mathrm{C}_{27} \mathrm{H}_{42} \mathrm{~N}_{2} \mathrm{O}_{7} \mathrm{NaS}[\mathrm{M}+\mathrm{Na}]^{+}$561.2610, found 561.2603.

4.2.6. tert-Butyl $[(2 S)$-2,3-dihydroxypropyl]3-\{[(2R)-2,3-dihydroxypropyl]thio\}-\{2-[(benzyloxy)imino]propyl\}carbamate (15)

To a solution of $(S, R)-14(0.050 \mathrm{~g}, 0.093 \mathrm{mmol})$ in a mixture of acetone/water $(10: 1(\mathrm{v}: \mathrm{v}), 600 \mu \mathrm{L})$ was added Amberlyst ${ }^{\circledR} 15(0.023 \mathrm{~g})$. After stirring at reflux for $4 \mathrm{~h}$, the solution was filtered on a Celite ${ }^{\circledR}$ pad and concentrated. The residue was purified by flash chromatography on silica gel using ethyl acetate as eluent to give pure $(S, R)-\mathbf{1 5}(0.014 \mathrm{~g}, 33 \%)$ as a colorless oil. ${ }^{1} \mathrm{H}$ NMR $\left(\mathrm{CDCl}_{3}\right)$ two isomers $E(\mathrm{~min})$ and $Z$ (maj) $\delta / \mathrm{ppm} 7.38-7.26$ (m, 10H, H-Ar, $Z$ and $E$ ), 5.08 (s, 2H, $\mathrm{CH}_{2} \mathrm{Ph}, E$ ), 5.07 (s, 2H, $\mathrm{CH}_{2} \mathrm{Ph}, Z$ ), 4.42 $\left(\mathrm{d}, J=16.0 \mathrm{~Hz}, 1 \mathrm{H}, \mathrm{CH}_{2} \mathrm{~S}, Z\right), 4.30-4.15\left(\mathrm{~m}, 3 \mathrm{H}, \mathrm{CH}_{2} \mathrm{~S}, Z\right.$ and $\left.E\right), 3.82\left(\mathrm{~m}, 2 \mathrm{H}, \mathrm{CHO}-\mathrm{CH}_{2} \mathrm{~N}, Z\right.$ and $\left.E\right)$, $3.73\left(\mathrm{Q}, J=6.0 \mathrm{~Hz}, 1 \mathrm{H}, \mathrm{CHO}-\mathrm{CH}_{2} \mathrm{~S}, Z\right), 3.68\left(\mathrm{Q}, J=5.5 \mathrm{~Hz}, 1 \mathrm{H}, \mathrm{CHO}-\mathrm{CH}_{2} \mathrm{~S}, E\right), 3.54-3.41(\mathrm{~m}, 10 \mathrm{H}$, $\mathrm{CH}_{2} \mathrm{O}, Z$ and $E, \mathrm{CH}_{2} \mathrm{~N}$ and $\left.\mathrm{NCH}_{2}\right), 3.37-3.30\left(\mathrm{~m}, 3 \mathrm{H}, \mathrm{CH}_{2} \mathrm{~N}\right.$ and $\left.\mathrm{NCH}_{2}\right), 3.28-3.10\left(\mathrm{~m}, 3 \mathrm{H}, \mathrm{CH}_{2} \mathrm{~N}\right.$ and $\left.\mathrm{NCH}_{2}\right), 2.67\left(\mathrm{~m}, 1 \mathrm{H}, \mathrm{SCH}_{2}, E\right), 2.56\left(\mathrm{~m}, 1 \mathrm{H}, \mathrm{SCH}_{2}, E\right), 2.56\left(\mathrm{dd},{ }^{2} J=14.0 \mathrm{~Hz},{ }^{3} J=7.0 \mathrm{~Hz}, 1 \mathrm{H}, \mathrm{SCH}_{2}, Z\right)$, $2.44\left(\mathrm{dd},{ }^{2} J=14.0 \mathrm{~Hz},{ }^{3} J=7.0 \mathrm{~Hz}, 1 \mathrm{H}, \mathrm{SCH}_{2}, Z\right) ;{ }^{13} \mathrm{C} \mathrm{NMR}\left(\mathrm{CD}_{3} \mathrm{OD}\right) \delta / \mathrm{ppm} 157.0$ and $156.8\left(\mathrm{CO}_{2} t-\mathrm{Bu}, Z\right)$, $155.6(\mathrm{C}=\mathrm{N}, Z), 155.4(\mathrm{C}=\mathrm{N}, E), 153.8$ and $153.7\left(\mathrm{CO}_{2} t-\mathrm{Bu}, E\right), 139.3(\mathrm{C}-\mathrm{Ar}, E), 139.1(\mathrm{C}-\mathrm{Ar}, Z)$, 129.4 (C-Ar, $Z$ and $E$ ), 129.2 (C-Ar, $Z$ ), 129.1 (C-Ar, E), 128.9 (C-Ar, $Z$ and $E$ ), 81.8 and $81.7(\mathrm{C}-$ $\left.\left(\mathrm{CH}_{3}\right)_{3}\right), 77.2\left(\mathrm{CH}_{2} \mathrm{Ph}, E\right), 77.1\left(\mathrm{CH}_{2} \mathrm{Ph}, \mathrm{Z}\right), 72.3\left(\mathrm{SCH}_{2}-\mathrm{CHO}, \mathrm{Z}\right), 72.2\left(\mathrm{SCH}_{2}-\mathrm{CHO}, E\right), 71.8$ and 71.7 $\left(\mathrm{NCH}_{2}-\mathrm{CHO}\right), 66.1\left(\mathrm{SCH}_{2} \mathrm{CH}-\mathrm{CH}_{2} \mathrm{O}, \mathrm{E}\right), 66.0\left(\mathrm{SCH}_{2} \mathrm{CH}-\mathrm{CH}_{2} \mathrm{O}, \mathrm{Z}\right), 65.4\left(\mathrm{NCH}_{2} \mathrm{CH}-\mathrm{CH}_{2} \mathrm{O}, E\right), 65.1$ $\left(\mathrm{NCH}_{2} \mathrm{CH}-\mathrm{CH}_{2} \mathrm{O}, \mathrm{Z}\right), 53.2\left(\mathrm{NCH}_{2} \mathrm{CH}-\mathrm{CH}_{2} \mathrm{O}, E\right), 52.4\left(\mathrm{NCH}_{2} \mathrm{CH}-\mathrm{CH}_{2} \mathrm{O}, \mathrm{Z}\right), 51.1$ and $50.8\left(\mathrm{~N}=\mathrm{CCH}_{2} \mathrm{~S}\right)$, 
46.2 and $45.8\left(\mathrm{~N}=\mathrm{CCH}_{2} \mathrm{~S}\right), 36.9$ and $36.7\left(\mathrm{SCH}_{2}-\mathrm{CHO}, E\right), 35.5$ and $35.3\left(\mathrm{~N}=\mathrm{CCH}_{2} \mathrm{~N}, Z\right), 33.5$ and $33.1\left(\mathrm{~N}=\mathrm{CH}_{2}, Z\right), 28.7\left(\mathrm{CH}_{3}\right), 26.6$ and $26.2\left(\mathrm{CH}_{3}\right)$.

4.2.7. (2E)- and (2Z)-1-(\{[(4S)-2,2-Dimethyl-1,3-dioxolan-4-yl]methyl\}amino)-3-\{[(4S)-2,2-dimethyl-1,3-dioxolan-4yl]methoxy\}propanone $O$-benzyloxime (16)

To a solution of $(S)-11 \mathrm{a}(0.166 \mathrm{~g} ; 0.51 \mathrm{mmol})$ in anhydrous methanol $(1.5 \mathrm{~mL})$ was added a solution of amine $(S)-9 \mathrm{c}(0.200 \mathrm{~g} ; 1.53 \mathrm{mmol})$ in methanol $(0.5 \mathrm{~mL})$. The resulting mixture was heated at reflux for $4 \mathrm{~h}$. The solvent was then concentrated and a saturated aqueous $\mathrm{NaHCO}_{3}(10 \mathrm{~mL})$ solution was added. The mixture was extracted with dichloromethane $(3 \times 10 \mathrm{~mL})$, dried $\left(\mathrm{MgSO}_{4}\right)$, and concentrated in vacuo. The crude product was purified by flash chromatography using (7:3) ethyl acetate/cyclohexane as eluent to give 16 as a yellow oil $(0.132 \mathrm{~g}, 61 \%)$. IR $v_{\max }\left(\right.$ neat, $\left.\mathrm{cm}^{-1}\right) 3345(\mathrm{~N}-\mathrm{H}), 1590(\mathrm{C}=\mathrm{N}), 1255-1050(\mathrm{C}-$ $\mathrm{O}) ;{ }^{1} \mathrm{H}$ NMR $\left(\mathrm{CDCl}_{3}\right) \delta / \mathrm{ppm} 7.35-7.26(\mathrm{~m}, 10 \mathrm{H}, \mathrm{H}-\mathrm{Ar}, E$ and $Z), 5.09\left(\mathrm{~s}, 2 \mathrm{H}, \mathrm{CH}_{2} \mathrm{Ph}, E\right), 5.06(\mathrm{~s}, 2 \mathrm{H}$, $\left.\mathrm{CH}_{2} \mathrm{Ph}, Z\right), 4.43\left(\mathrm{~d},{ }^{2} \mathrm{~J}=15.5 \mathrm{~Hz}, 1 \mathrm{H}, \mathrm{O}-\mathrm{CH}_{2}-\mathrm{C}=\mathrm{N}, Z\right), 4.40\left(\mathrm{~d},{ }^{2} J=15.5 \mathrm{~Hz}, 1 \mathrm{H}, \mathrm{O}-\mathrm{CH} H_{2}-\mathrm{C}=\mathrm{N}, Z\right)$, $4.27-4.15(\mathrm{~m}, 4 \mathrm{H}, \mathrm{H}-\mathrm{CHO}, E$ and $Z), 4.14\left(\mathrm{~d},{ }^{2} J=12.0 \mathrm{~Hz}, 1 \mathrm{H}, \mathrm{O}-\mathrm{CH}_{2}-\mathrm{C}=\mathrm{N}, E\right), 4.12\left(\mathrm{~d},{ }^{2} J=12.0 \mathrm{~Hz}\right.$, $\left.1 \mathrm{H}, \mathrm{O}-\mathrm{CH}_{2}-\mathrm{C}=\mathrm{N}, E\right), 4.05-3.96\left(\mathrm{~m}, 4 \mathrm{H}, \mathrm{CHO}-\mathrm{CH}_{2} \mathrm{O}, Z\right.$ and $\left.E\right), 3.72\left(\mathrm{dd},{ }^{2} J^{\prime}=8.0 \mathrm{~Hz},{ }^{3} \mathrm{~J}=6.5 \mathrm{~Hz}, 1 \mathrm{H}\right.$, $\left.\mathrm{O}-\mathrm{CH}_{2}-\mathrm{CHO}, \mathrm{Z}\right), 3.67\left(\mathrm{dd},{ }^{2} \mathrm{~J}=8.5 \mathrm{~Hz},{ }^{3} \mathrm{~J}=6.5 \mathrm{~Hz}, 1 \mathrm{H}, \mathrm{O}-\mathrm{CH}_{2}-\mathrm{CHO}, E\right), 3.62\left(\mathrm{dd},{ }^{2} \mathrm{~J}=8.0 \mathrm{~Hz},{ }^{3} \mathrm{~J}=7.0 \mathrm{~Hz}\right.$, $2 \mathrm{H}, \mathrm{NHCH}_{2} \mathrm{CH}-\mathrm{CH}_{2} \mathrm{O}, E$ and $\left.Z\right), 3.58\left(\mathrm{~d},{ }^{2} \mathrm{~J}=15.0 \mathrm{~Hz}, 1 \mathrm{H}, \mathrm{CH}_{2} \mathrm{NH}, E\right), 3.57\left(\mathrm{~d},{ }^{2} \mathrm{~J}=15.0 \mathrm{~Hz}, 1 \mathrm{H}, \mathrm{CH}_{2} \mathrm{NH}\right.$, $E), 3.53-3.40\left(\mathrm{~m}, 6 \mathrm{H}, \mathrm{CH}-\mathrm{O}-\mathrm{CH}_{2} \mathrm{C}=\mathrm{N}, E\right.$ and $\left.Z,=\mathrm{C}-\mathrm{CH}_{2} \mathrm{NH}, Z\right), 2.72-2.62\left(\mathrm{~m}, 4 \mathrm{H}, \mathrm{NH}-\mathrm{CH}_{2}, Z\right.$ and $E$ ), 1.91 (br s, 2H, NH, $E$ and $Z$ ), 1.40 and1.38 (s, $12 \mathrm{H}, \mathrm{Me}, Z$ and $E$ ), 1.34 and 1.33 (s, $12 \mathrm{H}, \mathrm{Me}, Z$ and $E) ;{ }^{13} \mathrm{C}$ NMR $\left(\mathrm{CDCl}_{3}\right) \delta / \mathrm{ppm} 156.9(\mathrm{C}=\mathrm{N}, Z), 156.6(\mathrm{C}=\mathrm{N}, E), 137.6(\mathrm{C}-\mathrm{Ar}, Z), 137.5(\mathrm{C}-\mathrm{Ar}, E)$, $128.3(\mathrm{C}-\mathrm{Ar}, Z$ and $E), 128.0(\mathrm{C}-\mathrm{Ar}, Z$ and $E), 127.8$ (C-Ar, $Z$ and $E), 109.4\left(\mathrm{C}-\left(\mathrm{CH}_{3}\right)_{2}, Z\right.$ and $\left.E\right), 109.0$ $\left(\mathrm{C}-\left(\mathrm{CH}_{3}\right)_{2}, Z\right.$ and $\left.E\right), 76.0\left(\mathrm{CH}_{2} \mathrm{Ph}, Z\right.$ and $\left.E\right), 75.3\left(\mathrm{NHCH}_{2} \mathrm{CHO}, Z\right), 75.1\left(\mathrm{NHCH}_{2} C H O, E\right), 74.4$

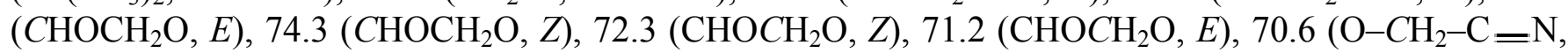
$E), 67.5\left(\mathrm{NHCH}_{2} \mathrm{CHOCH}_{2}, Z\right), 67.4\left(\mathrm{NHCH}_{2} \mathrm{CHOCH}_{2}, E\right), 66.6\left(\mathrm{OHCH}_{2} \mathrm{CHOCH}_{2}, E\right), 66.5$ $\left(\mathrm{OHCH}_{2} \mathrm{CHOCH}_{2}, Z\right), 66.3\left(\mathrm{OCH}_{2} \mathrm{C}=\mathrm{N}, Z\right), 52.2\left(\mathrm{NH}-\mathrm{CH}_{2}-\mathrm{CHO}, E\right), 51.5\left(\mathrm{NH}-\mathrm{CH}_{2}-\mathrm{CHO}, Z\right), 49.1$ $\left(\mathrm{N}=\mathrm{C}-\mathrm{CH}_{2}-\mathrm{NH}, Z\right), 44.9\left(\mathrm{~N}=\mathrm{C}-\mathrm{CH}_{2}-\mathrm{NH}, E\right), 26.9\left(\mathrm{CH}_{3}, Z\right), 26.8\left(\mathrm{CH}_{3}, E\right), 26.8\left(\mathrm{CH}_{3}, Z\right.$ and $\left.E\right), 25.4$ $\left(\mathrm{CH}_{3}, Z\right.$ and $\left.E\right), 25.3\left(\mathrm{CH}_{3}, Z\right.$ and $\left.E\right)$; HRMS (ESI): calculated for $\mathrm{C}_{22} \mathrm{H}_{35} \mathrm{~N}_{2} \mathrm{O}_{6}[\mathrm{M}+\mathrm{H}]^{+} 423.2495$, found 423.2497.

4.2.8. (2E)- and (2Z)-1-(\{[(4S)-2,2-Dimethyl-1,3-dioxolan-4-yl]methyl\}amino)-3-(\{[(4R)-2,2-dimethyl-1,3-dioxolan-4yl]methyl\}thio)propanone $O$-benzyloxime (17)

To a solution of $(R)-11 b(0.150 \mathrm{~g}, 0.44 \mathrm{mmol})$ in $1 \mathrm{~mL}$ of anhydrous methanol was added a solution of amine $(S)-9 \mathrm{c}(0.172 \mathrm{~g} ; 1.31 \mathrm{mmol})$ in methanol $(0.5 \mathrm{~mL})$. The resulting mixture was stirred under reflux for $8 \mathrm{~h}$. The solution was then concentrated in vacuo and a saturated aqueous solution of $\mathrm{NaHCO}_{3}(7 \mathrm{~mL})$ followed by dichloromethane $(15 \mathrm{~mL})$ was introduced. After extraction, the organic layer was dried $\left(\mathrm{MgSO}_{4}\right)$ and concentrated. The crude product was then purified by flash chromatography using $(1: 1 \rightarrow 7: 3)$ ethyl acetate/cyclohexane as eluent to give pure 17 as a yellow oil $(0.128 \mathrm{mg} ; 67 \%)$. Compound 17 appeared as a mixture of $Z$ and $E$ isomers. IR $v_{\max }$ (neat, $\left.\mathrm{cm}^{-1}\right) 3341(\mathrm{~N}-\mathrm{H}), 1624(\mathrm{C}=\mathrm{N})$, 1250-1050 (C-O); ${ }^{1} \mathrm{H}$ NMR $\left(\mathrm{CDCl}_{3}\right) \delta / \mathrm{ppm} 7.36-7.27(\mathrm{~m}, 10 \mathrm{H}, \mathrm{H}-\mathrm{Ar}, Z$ and $E), 5.08\left(\mathrm{~s}, 4 \mathrm{H}, \mathrm{CH}_{2} \mathrm{Ph}, Z\right.$ and $E), 4.24-4.14(\mathrm{~m}, 4 \mathrm{H}, \mathrm{CHO}, Z$ and $E), 4.00\left(\mathrm{dd},{ }^{2} J=8.0 \mathrm{~Hz},{ }^{3} \mathrm{~J}=6.5 \mathrm{~Hz}, 1 \mathrm{H}, \mathrm{NCH}_{2} \mathrm{CHCH}_{2} \mathrm{O}, Z\right), 4.02-$ $3.96\left(\mathrm{~m}, 2 \mathrm{H}, \mathrm{NCH}_{2} \mathrm{CHCH}_{2} \mathrm{O}, E\right.$ and $\left.\mathrm{SCH}_{2} \mathrm{CHCH}_{2} \mathrm{O}, E\right), 3.95\left(\mathrm{dd},{ }^{2} J=8.5 \mathrm{~Hz},{ }^{3} J=6.0 \mathrm{~Hz}, 1 \mathrm{H}\right.$, $\left.\mathrm{SCH}_{2} \mathrm{CHCH}_{2} \mathrm{O}, Z\right), 3.67-3.55\left(\mathrm{~m}, 6 \mathrm{H}, \mathrm{SCH}_{2} \mathrm{CHCH}_{2} \mathrm{O}(\mathrm{Z}\right.$ and $E), \mathrm{NCH}_{2} \mathrm{CHCH}_{2} \mathrm{O}(\mathrm{Z}$ and $E), \mathrm{N}=\mathrm{CCH}_{2} \mathrm{~N}$, $E), 3.48\left(\mathrm{~s}, 2 \mathrm{H}, \mathrm{N}=\mathrm{CCH}_{2} \mathrm{~N}, Z\right), 3.46\left(\mathrm{~s}, 2 \mathrm{H}, \mathrm{N}=\mathrm{CCH}_{2} \mathrm{~N}, Z\right), 3.32\left(\mathrm{~d},{ }^{2} J=13.5 \mathrm{~Hz}, 1 \mathrm{H}, \mathrm{SCH}_{2} \mathrm{C}=\mathrm{N}, E\right)$, $3.29\left(\mathrm{~d},{ }^{2} J=13.5 \mathrm{~Hz}, 1 \mathrm{H}, \mathrm{SCH}_{2} \mathrm{C}=\mathrm{N}, E\right), 2.70\left(\mathrm{dd},{ }^{2} J=13.5 \mathrm{~Hz},{ }^{3} J=6.5 \mathrm{~Hz}, 1 \mathrm{H}, \mathrm{OCHCH}_{2} \mathrm{~S}, Z\right), 2.67(\mathrm{~d}$, ${ }^{3} J=6.0 \mathrm{~Hz}, 4 \mathrm{H}, \mathrm{NHCH}_{2} \mathrm{CHO}, E$ and $\left.Z\right), 2.59\left(\mathrm{dd},{ }^{2} J=13.5 \mathrm{~Hz},{ }^{3} J=6.5 \mathrm{~Hz}, 1 \mathrm{H}, \mathrm{OCHCH}_{2} \mathrm{~S}, E\right), 2.56(\mathrm{dd}$, $\left.{ }^{2} J=13.5 \mathrm{~Hz},{ }^{3} \mathrm{~J}=6.5 \mathrm{~Hz}, 1 \mathrm{H}, \mathrm{OCHCH}_{2} \mathrm{~S}, Z\right), 2.47\left(\mathrm{dd},{ }^{2} J=13.5 \mathrm{~Hz},{ }^{3} \mathrm{~J}=6.5 \mathrm{~Hz}, 1 \mathrm{H}, \mathrm{OCHCH}_{2} \mathrm{~S}, E\right), 1.64$ (br s, $2 \mathrm{H}, \mathrm{NH}, E$ and $Z$ ), 1.41 (s, 6H, Me, $E$ and $Z$ ), 1.40 (s, 6H, Me, $E$ and $Z$ ), 1.34 (s, 6H, Me, $E$ and $Z$ ), 1.32 $(\mathrm{s}, 6 \mathrm{H}, \mathrm{Me}, E$ and $Z) ;{ }^{13} \mathrm{C} \mathrm{NMR}\left(\mathrm{CDCl}_{3}\right) \delta / \mathrm{ppm} 156.5(\mathrm{C}=\mathrm{N}, E), 155.8(\mathrm{C}=\mathrm{N}, Z), 137.7(\mathrm{C}-\mathrm{Ar}, E)$, 137.4 (C-Ar, Z), 128.3 (C-Ar, $Z$ and $E), 128.0$ (C-Ar, $Z$ and $E), 127.9$ (C-Ar, Z), 127.8 (C-Ar, E), 109.4 $\left(\mathrm{C}-\left(\mathrm{CH}_{3}\right)_{2}, Z\right.$ and $\left.E\right), 109.1\left(\mathrm{C}-\left(\mathrm{CH}_{3}\right)_{2}, Z\right.$ and $\left.E\right), 76.0\left(\mathrm{CH}_{2} \mathrm{Ph}, Z\right.$ and $\left.E\right), 75.2\left(\mathrm{NHCH}_{2}-C H O, Z\right), 75.1$ $\left(\mathrm{NHCH}_{2}-\mathrm{CHO}, E\right), 74.8\left(\mathrm{SCH}_{2}-\mathrm{CHO}, \mathrm{Z}\right.$ and $\left.E\right), 68.6\left(\mathrm{SCH}_{2} \mathrm{CHCH}_{2} \mathrm{O}, Z\right.$ and $\left.E\right), 67.4\left(\mathrm{NHCH}_{2} \mathrm{CHCH}_{2} \mathrm{O}\right.$, $Z), 67.3\left(\mathrm{NHCH}_{2} \mathrm{CHCH}_{2} \mathrm{O}, E\right), 52.1\left(\mathrm{NH}-\mathrm{CH}_{2}-\mathrm{CHO}, E\right), 51.5\left(\mathrm{NH}-\mathrm{CH}_{2}-\mathrm{CHO}, Z\right), 50.2\left(\mathrm{~N}=\mathrm{C}-C \mathrm{H}_{2}, Z\right)$, $45.0\left(\mathrm{~N}=\mathrm{C}-\mathrm{CH}_{2}, E\right), 35.2\left(\mathrm{~S}-\mathrm{CH}_{2}-\mathrm{CHO}, Z\right), 33.8\left(\mathrm{~S}-\mathrm{CH}_{2}-\mathrm{CHO}, E\right), 33.2\left(\mathrm{~S}-\mathrm{CH}_{2}-\mathrm{C}=\mathrm{N}, E\right), 26.9$ $\left(\mathrm{CH}_{3}, E\right), 26.8\left(\mathrm{CH}_{3}, Z\right), 25.6\left(\mathrm{CH}_{3}, E\right), 25.4\left(\mathrm{CH}_{3}, Z\right), 25.5\left(\mathrm{CH}_{3}, Z\right.$ and $\left.E\right), 25.3\left(\mathrm{~S}-\mathrm{CH}_{2}-\mathrm{C}=\mathrm{N}, Z\right)$; HRMS (ESI): calculated for $\mathrm{C}_{22} \mathrm{H}_{35} \mathrm{~N}_{2} \mathrm{O}_{5} \mathrm{~S}[\mathrm{M}+\mathrm{H}]^{+} 439.2267$, found 439.2251.

4.2.9. $\quad N$-\{[(4S)-2,2-Dimethyl-1,3-dioxolan-4-yl]methyl\}- $N$-3-\{[(4S)-2,2-dimethyl-1,3-dioxolan-4-yl]methoxy\}-2$\{[($ benzyloxy)imino]propyl\}benzamide (18) 
To $(S, S)-16(0.317 \mathrm{~g}, 0.75 \mathrm{mmol})$ in anhydrous dichloromethane $(8 \mathrm{~mL})$ were added at $0{ }^{\circ} \mathrm{C}$ and under argon, triethylamine $(155 \mu \mathrm{L}, 1.13 \mathrm{mmol})$ and 4-dimethylaminopyridine $(0.018 \mathrm{mg}, 0.15 \mathrm{mmol})$. After $15 \mathrm{~min}$, benzoylchloride $(105 \mu \mathrm{L}, 0.90 \mathrm{mmol})$ was added dropwise to the reaction mixture. The solution was quenched with $\mathrm{H}_{2} \mathrm{O}$ after $2 \mathrm{~h}$ and the mixture was extracted with dichloromethane. The combined organic extracts were dried over $\mathrm{MgSO}_{4}$, filtered, and concentrated in vacuo. The crude product was purified by silica gel chromatography using (3:2) cyclohexane/ethyl acetate as eluant to give $\mathbf{1 8}$ as a colorless oil $(0.355 \mathrm{~g}, 90 \%)$. Compound 18 is made of a mixture of the two isomers $Z$ and $E$. IR $v_{\max }$ (neat, $\left.\mathrm{cm}^{-1}\right) 1635(\mathrm{C}=\mathrm{O}), 1260-1000(\mathrm{C}-\mathrm{O}) ;{ }^{1} \mathrm{H}$ NMR $\left(\mathrm{CDCl}_{3}\right) \delta / \mathrm{ppm} 7.40-7.20(\mathrm{~m}, 10 \mathrm{H}, \mathrm{H}-\mathrm{Ar}), 5.16-$ $5.00\left(\mathrm{~m}, 2 \mathrm{H}, \mathrm{CH}_{2} \mathrm{Ph}\right), 4.69-3.75\left(\mathrm{~m}, 8 \mathrm{H}, \mathrm{CHO}, \mathrm{CH}_{2} \mathrm{O}, \mathrm{O}-\mathrm{CH}_{2}-\mathrm{C}=\right), 3.75-3.15\left(\mathrm{~m}, 6 \mathrm{H}, \mathrm{CHO}-\mathrm{CH}_{2}-\mathrm{O}\right.$, $\left.\mathrm{N}-\mathrm{CH}_{2}-\mathrm{CHO},=\mathrm{C}-\mathrm{CH}_{2}-\mathrm{N}\right), 1.40-1.20(\mathrm{~m}, 12 \mathrm{H}, \mathrm{Me}) ;{ }^{13} \mathrm{C} \mathrm{NMR}\left(\mathrm{CDCl}_{3}\right) \delta / \mathrm{ppm} 172.6(\mathrm{C}=, E$ and $Z)$, $153.8(\mathrm{NCO}, E$ and $Z$ ), 137.7 (C-Ar-Bn, $E$ and $Z$ ), 136.2 (C-Ar, Z), 135.8 (C-Ar, E), 129.5 (C-Ar, E), 129.3 (C-Ar, Z), 128.3 (C-Ar-Bn, $E$ and $Z$ ), 128.2 (C-Ar, $E$ and $Z$ ), 128.0 (C-Ar-Bn, $E$ and $Z$ ), 127.0 $(\mathrm{C}-\mathrm{Ar}-\mathrm{Bn}, E$ and $Z), 126.4(\mathrm{C}-\mathrm{Ar}, E$ and $Z), 109.4\left(C-\left(\mathrm{CH}_{3}\right)_{2}, Z\right), 109.1\left(C-\left(\mathrm{CH}_{3}\right)_{2}, E\right), 76.4\left(C \mathrm{H}_{2}-\mathrm{Ph}\right.$, $Z), 76.2\left(\mathrm{CH}_{2}-\mathrm{Ph}, E\right), 75.1\left(\mathrm{NCH}_{2}-\mathrm{CHO}, Z\right), 74.4\left(\mathrm{NCH}_{2}-\mathrm{CHO}, E\right), 74.2\left(\mathrm{OCH}_{2}-C \mathrm{HO}, E\right.$ and $\left.Z\right), 72.5$ $\left(\mathrm{CHO}-\mathrm{CH}_{2}-\mathrm{O}, \mathrm{E}\right), 72.0\left(\mathrm{CHO}-\mathrm{CH}_{2}-\mathrm{O}, \mathrm{Z}\right), 67.4-66.9-66.6-66.3-65.8-65.5\left(\mathrm{CH}_{2}-\mathrm{O}, \mathrm{O}-\mathrm{CH}_{2}-\mathrm{C}=, E\right.$ and $Z), 51.8\left(\mathrm{~N}-\mathrm{CH}_{2}-\mathrm{CHO}, E\right), 50.3\left(\mathrm{~N}-\mathrm{CH}_{2}-\mathrm{CHO}, Z\right), 48.1\left(=\mathrm{C}-\mathrm{CH}_{2}-\mathrm{N}, Z\right), 44.6\left(=\mathrm{C}-\mathrm{CH}_{2}-\mathrm{N}, E\right)$, 26.8-26.7-26.6-25.4-25.3 $\left(\mathrm{CH}_{3}, E\right.$ and Z); HRMS (ESI): calculated for $\mathrm{C}_{29} \mathrm{H}_{38} \mathrm{~N}_{2} \mathrm{O}_{7} \mathrm{Na}[\mathrm{M}+\mathrm{Na}]^{+}$ 549.2577, found 549.2572.

4.2.10. $\quad N$-\{[(4S)-2,2-Dimethyl-1,3-dioxolan-4-yl]methyl\}- $N$-[3-(\{[(4R)-2,2-dimethyl-1,3-dioxolan-4-yl]methyl\}-thio)-2[(benzyloxy)imino]propyl]benzamide (19)

Compound $17(0.350 \mathrm{~g} ; 0.80 \mathrm{mmol})$ was dissolved in anhydrous dichloromethane $(8 \mathrm{~mL})$ at $0{ }^{\circ} \mathrm{C}$ and under argon. Triethylamine $(170 \mu \mathrm{L}, 1.20 \mathrm{mmol})$ and 4-dimethylaminopyridine $(0.020 \mathrm{~g}, 0.16 \mathrm{mmol})$ were added followed by benzoylchloride $(110 \mu \mathrm{L}, 0.96 \mathrm{mmol})$. The mixture was stirred at $0{ }^{\circ} \mathrm{C}$ for $2 \mathrm{~h}$ whereupon the reaction was stopped by addition of water, extracted with ethyl acetate, and dried $\left(\mathrm{MgSO}_{4}\right)$. After evaporation of the solvent, the crude product was purified by flash chromatography using (7:3) cyclohexane/ethyl acetate as eluent to give pure $19(0.412 \mathrm{mg}, 95 \%)$ as a colorless oil. IR $v_{\max }$ (neat, $\left.\mathrm{cm}^{-1}\right) 1638(\mathrm{C}=\mathrm{O}), 1260-1060(\mathrm{C}-\mathrm{O}) ;{ }^{1} \mathrm{H}$ NMR $\left(\mathrm{CDCl}_{3}\right) \delta / \mathrm{ppm} 7.40-7.26(\mathrm{~m}, 10 \mathrm{H}, \mathrm{H}-\mathrm{Ar}), 5.09(\mathrm{~s}, 2 \mathrm{H}$, $\left.\mathrm{CH}_{2} \mathrm{Ph}\right), 4.67-3.74\left(\mathrm{~m}, 6 \mathrm{H}, \mathrm{CHO}, \mathrm{CH}_{2} \mathrm{O}\right), 3.73-3.00\left(\mathrm{~m}, 6 \mathrm{H}, \mathrm{N}-\mathrm{CH}_{2}-\mathrm{CHO}, \mathrm{S}-\mathrm{CH}_{2}-\mathrm{C}=\mathrm{N}\right.$ and $\mathrm{N}=\mathrm{C}-$ $\left.\mathrm{CH}_{2} \mathrm{~N}\right), 2.82-2.20\left(\mathrm{~m}, 2 \mathrm{H}, \mathrm{CHO}-\mathrm{CH}_{2}-\mathrm{S}\right), 1.45-1.20(\mathrm{~m}, 12 \mathrm{H}, \mathrm{Me}) ;{ }^{13} \mathrm{C} \mathrm{NMR}\left(\mathrm{CDCl}_{3}\right) \delta / \mathrm{ppm} 172.5(\mathrm{C}=$ $\mathrm{N}, E), 172.4(\mathrm{C}=\mathrm{N}, Z), 152.1(\mathrm{C}=\mathrm{O}, E$ and $Z), 137.3(\mathrm{COC}-\mathrm{Ar}, E$ and $Z), 135.9$ (COC-Ar, Z), 135.7 (COC-Ar, E), 129.6 (COC-Ar, E), 129.4 (COC-Ar, Z), 128.6, 128.3, 128.2, 128.1, 128.0, 127.9, 127.0 and $126.5(\mathrm{C}-\mathrm{Ar}, E$ and $Z), 109.4\left(\mathrm{C}-\left(\mathrm{CH}_{3}\right)_{2}, Z\right), 109.2\left(\mathrm{C}-\left(\mathrm{CH}_{3}\right)_{2}, E\right), 76.4\left(\mathrm{CH}_{2} \mathrm{Ph}, E\right), 76.3\left(\mathrm{CH}_{2} \mathrm{Ph}, Z\right)$, 74.9, 74.8 and $74.6(\mathrm{CHO}, E$ and $Z), 68.6\left(\mathrm{CH}_{2}-\mathrm{CHOCH}_{2} \mathrm{~S}, E\right), 68.5\left(\mathrm{CH}_{2}-\mathrm{CHOCH}_{2} \mathrm{~S}, \mathrm{Z}\right), 67.2\left(\mathrm{CH}_{2}-\right.$ $\left.\mathrm{CHOCH}_{2} \mathrm{~N}, Z\right), 66.8\left(\mathrm{CH}_{2}-\mathrm{CHOCH}_{2} \mathrm{~N}, E\right), 51.6\left(\mathrm{~N}-\mathrm{CH}_{2}-\mathrm{CHO}, Z\right), 47.9\left(\mathrm{~N}=\mathrm{CCH}_{2}, Z\right), 46.1\left(\mathrm{~N}-\mathrm{CH}_{2}-\right.$ $\mathrm{CHO}, E), 42.6\left(\mathrm{~N}=\mathrm{CCH}_{2}, E\right), 35.5\left(\mathrm{CHO}-\mathrm{CH}_{2} \mathrm{~S}, E\right), 34.7\left(\mathrm{CHO}-\mathrm{CH}_{2} \mathrm{~S}, \mathrm{Z}\right), 34.1$ and $33.4\left(\mathrm{~S}-\mathrm{CH}_{2}-\mathrm{C}=\right.$ $\mathrm{N}, E), 26.8\left(\mathrm{CH}_{3}, E\right.$ and $\left.Z\right), 26.0\left(\mathrm{~S}-\mathrm{CH}_{2}-\mathrm{C}=\mathrm{N}, Z\right), 25.4\left(\mathrm{CH}_{3}, E\right.$ and $\left.Z\right)$; HRMS (ESI): calculated for $\mathrm{C}_{29} \mathrm{H}_{38} \mathrm{~N}_{2} \mathrm{O}_{6} \mathrm{NaS}[\mathrm{M}+\mathrm{Na}]^{+}$565.2348, found 565.2374.

4.2.11. (2R,8S)-2,8-Dihydroxymethyl-10-benzoyl-10-aza-1,4,7-trioxaspiro[5.5]undecane (20)

A solution of $(S, S)-18(0.150 \mathrm{~g}, 0.28 \mathrm{mmol})$ in $2.8 \mathrm{~mL}$ of a $10: 1(\mathrm{v} / \mathrm{v})$ mixture of acetone/water and Amberlyst ${ }^{\circledR} 15(0.070 \mathrm{~g})$ was heated for 2 days under reflux with $2.8 \mathrm{mmol}$ of paraformaldehyde. After cooling and filtration on a Celite ${ }^{\circledR}$ pad, the solvent was evaporated in vacuo and the crude mixture was purified by flash chromatography using (49:1) ethyl acetate/ $\mathrm{MeOH}$ as eluent to give a mixture of isomers 20 as a colorless oil $(0.056 \mathrm{~g}, 61 \%)$. IR $v_{\max }$ (neat, $\left.\mathrm{cm}^{-1}\right) 3400(\mathrm{O}-\mathrm{H}), 1618(\mathrm{C}=\mathrm{O}), 1051(\mathrm{C}-\mathrm{O}) ;{ }^{13} \mathrm{C}$ NMR (DMSO- $\left.d_{6}, 293 \mathrm{~K}\right) \delta / \mathrm{ppm}$ 170.6-170.4-170.0-169.6 (C-Ar), 136.7-135.3 (C-Ar), 129.7-129.4 (C-Ar), 128.4-128.2 (C-Ar), 127.3-127.0-126.8 (C-Ar), 92.1-91.6 (C-6), 73.4-73.2-71.9-69.0-67.867.4 (C-3 and C-5), 72.8-72.4-70.5-70.3-68.6 (C-2 and C-8), 63.0-62.9-62.8-61.9-61.6-61.0 (C-12 and C-13), 52.2-50.7-50.3-48.6-46.4-45.1-44.9-43.4 (C-9 and C-11); HRMS (ESI): calculated for $\mathrm{C}_{16} \mathrm{H}_{21} \mathrm{NO}_{6} \mathrm{Na}[\mathrm{M}+\mathrm{Na}]^{+} 346.1267$, found 346.1261 .

4.2.12. (2R,8S)-2,8-Dihydroxymethyl-10-benzoyl-10-aza-4-thia-1,7-dioxaspiro[5.5] undecane (21)

A solution of oxime $(R, S)-19(0.112 \mathrm{~g}, 0.20 \mathrm{mmol})$ in $2 \mathrm{~mL}$ of a $10: 1(\mathrm{v} / \mathrm{v})$ mixture of acetone/water with Amberlyst ${ }^{\circledR} 15(0.050 \mathrm{~g})$ and $2 \mathrm{mmol}$ of paraformaldehyde was heated under reflux for 2 days. After cooling and filtration on a Celite ${ }^{\circledR}$ pad, the solvent was evaporated in vacuo and the crude mixture purified by silica gel chromatography using $(1: 0 \rightarrow 49: 1)$ ethyl acetate/ $\mathrm{MeOH}$ as eluent to give 21 as a white syrup $(0.045 \mathrm{~g}, 65 \%)$. IR $v_{\max }\left(\right.$ neat, $\left.\mathrm{cm}^{-1}\right) 3394(\mathrm{O}-\mathrm{H}), 1618(\mathrm{C}=\mathrm{O}), 1047(\mathrm{C}-\mathrm{O})$; ${ }^{13} \mathrm{C}$ NMR $\left(\mathrm{DMSO}-d_{6}, 293 \mathrm{~K}\right) \delta / \mathrm{ppm}$ 170.1-169.4 (N-CO), 135.7 (C-Ar), 129.4 (C-Ar), 128.4-128.2 (C-Ar), 
127.3-126.6 (C-Ar), 91.6-91.2 (C-6), 70.3-70.2 (C-2), 68.7 (C-8), 63.9-63.8-61.9-61.5 (C-12 and C13), 54.1-48.5-43.5 (C-9 and C-11), 30.8-30.5 (C-5), 26.6 (C-3); HRMS (ESI): calculated for $\mathrm{C}_{16} \mathrm{H}_{21} \mathrm{NO}_{5} \mathrm{NaS}[\mathrm{M}+\mathrm{Na}]^{+}$362.1038, found 362.1021.

\subsubsection{2,8-Dihydroxymethyl-10-benzoyl-10-aza-1,4,7-trioxaspiro[5.5]undecane (22)}

To a solution of spiroketal $20(0.056 \mathrm{~g}, 0.17 \mathrm{mmol})$ in THF $(1 \mathrm{~mL})$ was added at $0{ }^{\circ} \mathrm{C}$ and under argon, a $1.0 \mathrm{M}$ solution of $\mathrm{LiAlH}_{4}$ in THF $(693 \mu \mathrm{L}, 0.68 \mathrm{mmol})$. After $3 \mathrm{~h}$, the reaction is quenched by adjunction of water and the residue filtered over $\mathrm{MgSO}_{4}$. The solvent was eliminated to give nearly quantitatively a crude and inseparable mixture of isomers 22 , in a $4: 1$ ratio as determined from ${ }^{13} \mathrm{C}$ NMR. ${ }^{13} \mathrm{C}$ NMR $\left(\mathrm{CDCl}_{3}\right) \delta / \mathrm{ppm}$ 105.9-91.6 (C-6), 75.0-70.0 (C-8), 72.6-70.0 (C-5), 70.6-69.0 (C-2), 68.4-67.2 (C-3), 63.7-63.4 ( $\left.\mathrm{CH}_{2} \mathrm{OH}\right), 62.5-62.0\left(\mathrm{CH}_{2} \mathrm{OH}\right), 49.9-49.1$ (C-11), 47.9-46.1 (C-9).

Major isomer: $(2 R, 6 S, 8 S)-22 \mathrm{a}:{ }^{1} \mathrm{H}$ NMR $\left(\mathrm{CDCl}_{3}\right) \delta / \mathrm{ppm} 4.06(\mathrm{~m}, 1 \mathrm{H}, \mathrm{H}-2), 3.90(\mathrm{~m}, 1 \mathrm{H}, \mathrm{H}-8), 3.60-3.48$ $\left(\mathrm{m}, 4 \mathrm{H}, \mathrm{CH}_{2} \mathrm{OH}\right), 3.74\left(\mathrm{dd}, J_{3 \mathrm{eq} 3 \mathrm{ax}}=11.0 \mathrm{~Hz}, J_{3 \mathrm{eq} 2 \mathrm{ax}}=2.5 \mathrm{~Hz}, 1 \mathrm{H}, \mathrm{H}-3_{\mathrm{eq}}\right), 3.60\left(\mathrm{~d},{ }^{2} J=11.5 \mathrm{~Hz}, 1 \mathrm{H}, \mathrm{H}-5\right)$, $3.33\left(\mathrm{t}, J_{3 \mathrm{ax} 3 \mathrm{eq}}=J_{3 \mathrm{ax} 2 \mathrm{ax}}=11.0 \mathrm{~Hz}, 1 \mathrm{H}, \mathrm{H}-3_{\mathrm{ax}}\right), 3.26\left(\mathrm{~d},{ }^{2} J=11.5 \mathrm{~Hz}, 1 \mathrm{H}, \mathrm{H}-5\right), 3.13(\mathrm{br} \mathrm{d}, 1 \mathrm{H}, \mathrm{NH}), 2.83$ (br d, $\left.J_{9 \mathrm{eq} 9 \mathrm{ax}}=12.0 \mathrm{~Hz}, 1 \mathrm{H}, \mathrm{H}-9_{\mathrm{eq}}\right), 2.73\left(\mathrm{~d},{ }^{2} J=13.0 \mathrm{~Hz}, 1 \mathrm{H}, \mathrm{H}-11\right), 2.57\left(\mathrm{t}, J_{9 \mathrm{ax} 9 \mathrm{eq}}=J_{9 \mathrm{ax} 8 \mathrm{ax}}=12.0 \mathrm{~Hz}, 1 \mathrm{H}, \mathrm{H}-9_{\mathrm{ax}}\right)$, $2.52\left(\mathrm{~d},{ }^{2} J=13.0 \mathrm{~Hz}, 1 \mathrm{H}, \mathrm{H}-11\right) ;{ }^{13} \mathrm{C} \mathrm{NMR}\left(\mathrm{CDCl}_{3}\right) \delta / \mathrm{ppm} 91.6(\mathrm{C}-6), 70.0(\mathrm{C}-8), 70.0$ (C-5), $69.0(\mathrm{C}-2)$, $67.2(\mathrm{C}-3), 63.4\left(\mathrm{CH}_{2} \mathrm{OH}\right), 62.0\left(\mathrm{CH}_{2} \mathrm{OH}\right), 49.1$ (C-11), 46.1 (C-9); HRMS (ESI): calculated for $\mathrm{C}_{9} \mathrm{H}_{18} \mathrm{NO}_{5}[\mathrm{M}+\mathrm{H}]^{+} 220.1185$, found 220.1175 .

\subsubsection{2,8-Dihydroxymethyl-10-benzoyl-10-aza-4-thia-1,7-dioxaspiro[5.5]undecane (23)}

To a solution of spiroketal $21(0.060 \mathrm{~g}, 0.18 \mathrm{mmol})$ in THF $(1 \mathrm{~mL})$ was added at $0{ }^{\circ} \mathrm{C}$ and under argon, a $1.0 \mathrm{M}$ solution of $\mathrm{LiAlH}_{4}$ in THF $(710 \mu \mathrm{L}, 0.72 \mathrm{mmol})$. After $3 \mathrm{~h}$, the reaction is quenched by adjunction of water and the residue filtered over $\mathrm{MgSO}_{4}$. The solvent was eliminated to give nearly quantitatively a crude and inseparable mixture of isomers 23, in a 9:1 ratio (determined from $\left.{ }^{13} \mathrm{C} N \mathrm{NMR}\right) .{ }^{13} \mathrm{C}$ NMR $\left(\mathrm{CDCl}_{3}\right) \delta / \mathrm{ppm}$ 99.9-90.9 (C-6), 71.4-70.8 (C-2), 70.8-70.2 (C-8), 68.8-63.3 $\left(\mathrm{CH}_{2} \mathrm{OH}\right), 65.2-65.2$ $\left(\mathrm{CH}_{2} \mathrm{OH}\right), 53.0-51.6(\mathrm{C}-11), 47.7-46.1(\mathrm{C}-9), 37.0^{*}-32.0(\mathrm{C}-5), 36.6^{*}-26.2(\mathrm{C}-3)$.

Major isomer: $(2 R, 6 S, 8 S)-23 a:{ }^{1} \mathrm{H}$ NMR $\left(\mathrm{CDCl}_{3}\right) \delta / \mathrm{ppm} 4.04(\mathrm{~m}, 1 \mathrm{H}, \mathrm{H}-2), 3.89(\mathrm{~m}, 1 \mathrm{H}, \mathrm{H}-8), 3.58-3.45$ $\left(\mathrm{m}, 4 \mathrm{H}, \mathrm{CH}_{2} \mathrm{OH}\right), 2.81$ (br d, $\left.J_{9 \mathrm{eq} 9 \mathrm{ax}}=12.0 \mathrm{~Hz}, 1 \mathrm{H}, \mathrm{H}-9_{\mathrm{eq}}\right), 2.79\left(\mathrm{~d},{ }^{2} J=13.0 \mathrm{~Hz}, 1 \mathrm{H}, \mathrm{H}-11\right), 2.67(\mathrm{~d}$, $\left.{ }^{2} J=13.5 \mathrm{~Hz}, 1 \mathrm{H}, \mathrm{H}-5\right), 2.57\left(\mathrm{~d},{ }^{2} J=13.0 \mathrm{~Hz}, 1 \mathrm{H}, \mathrm{H}-11\right), 2.51\left(\mathrm{t}, J_{9 \mathrm{ax} 9 \mathrm{eq}}=J_{9 \mathrm{ax} 8 \mathrm{ax}}=12.0 \mathrm{~Hz}, 1 \mathrm{H}, \mathrm{H}-9_{\mathrm{ax}}\right), 2.48(\mathrm{t}$, $\left.J_{3 \mathrm{ax} 3 \mathrm{eq}}=J_{3 \mathrm{ax} 2 \mathrm{ax}}=12.0 \mathrm{~Hz}, 1 \mathrm{H}, \mathrm{H}-3_{\mathrm{ax}}\right), 2.29\left(\mathrm{~d},{ }^{2} J=13.5 \mathrm{~Hz}, 1 \mathrm{H}, \mathrm{H}-5\right), 2.18\left(\right.$ br d, $\left.J_{3 \mathrm{eq} 3 \mathrm{ax}}=13.0 \mathrm{~Hz}, 1 \mathrm{H}, \mathrm{H}-3_{\mathrm{eq}}\right)$; ${ }^{13} \mathrm{C} \mathrm{NMR}\left(\mathrm{CDCl}_{3}\right) \delta / \mathrm{ppm} 90.9(\mathrm{C}-6), 70.8(\mathrm{C}-2), 70.2(\mathrm{C}-8), 65.2\left(\mathrm{CH}_{2} \mathrm{OH}\right), 63.3\left(\mathrm{CH}_{2} \mathrm{OH}\right), 53.0(\mathrm{C}-11)$, 46.1 (C-9), 32.0 (C-5), 26.2 (C-3); HRMS (ESI): calculated for $\mathrm{C}_{9} \mathrm{H}_{18} \mathrm{NO}_{4} \mathrm{~S}[\mathrm{M}+\mathrm{H}]^{+} 236.0957$, found 236.0946.

\section{References and notes}

1 (a)W. Francke and W. Kitching, Curr. Org. Chem. 5 (2001), pp. 233-251.

(b)M.F. Jacobs and W. Kitching, Curr. Org. Chem. 2 (1998), pp. 1617-1661.

(c)M.T. Fletcher and W. Kitching, Chem. Rev. 95 (1995), pp. 789-828.

(d)F. Perron and K.F. Albizati, Chem. Rev. 89 (1989), pp. 1617-1661.

2 For recent reviews on spiroketal syntheses see:.

(a)J.E. Aho, P.M. Pihko and T.K. Rissa, Chem. Rev. 105 (2005), pp. 4406-4440.

(b)K.T. Mead and B.N. Brewer, Curr. Org. Chem. 7 (2003), pp. 227-256.

(c)M.A. Brimble and D.P. Furkert, Curr. Org. Chem. 7 (2003), pp. 1-24.

(d)M.A. Brimble and F.A. Farès, Tetrahedron 55 (1999), pp. 7661-7706.

(e)J. Pietruszka, Angew. Chem., Int. Ed. 37 (1998), pp. 2629-2636.

(f)P. Cohen, C.F.B. Holmes and Y. Tsukitani, Trends Biochem. Sci. 15 (1990), pp. 98-102.

(g)P. Cohen, Annu. Rev. Biochem. 58 (1989), pp. 453-508.

3 (a)E.M. Seward, E. Carlson, T. Harrison, K.E. Haworth, R. Herbert, F.J. Kelleher, M.M. Kurtz, J. Moseley, S.N. Owen, A.P. Owens, S.J. Sadowski, C.J. Swain and B.J. Williams, Bioorg. Med. Chem. Lett. 12 (2002), pp. 2515-2518. (b) Moseley, J. D.; Swain, C. J. WO 97/30056, 1997.

4 T. Ercégovic and G. Magnusson, J. Org. Chem. 61 (1996), pp. 179-184.

(b)N. Hossain, A. Zapata, M. Wilstermann, U.J. Nilsson and G. Magnusson, Carbohydr. Res. 337 (2002), pp. $569-580$.

5 A. Bartolozzi, G. Capozzi, S. Menichetti and C. Nativi, Org. Lett. 2 (2000), pp. 251-253.

6

(a)B.L. Yin, Z.M. Yang, T.S. Hu and Y.L. Wu, Synthesis 13 (2003), pp. 1995-2000.

(b)B.L. Yin, Z.M. Yang, T.S. Hu and Y.L. Wu, ARKIVOC 2 (2003), pp. 70-83.

7 For synthesis based on a methylene strategy see:.

(a)N. Ardes-Guisot, B. Ouled-Lahoucine, I. Canet and M.E. Sinibaldi, Tetrahedron Lett. 48 (2007), pp. 8511-8513.

(b)J. Meléndez, F. Alonso and M. Yus, Tetrahedron Lett. 47 (2006), pp. 1187-1191.

(c)A.G.M. Barrett, D.C. Braddock, P.D. De Koning, A.J.P. White and D.J. Williams, J. Org. Chem. 65 (2000), pp. 375-380.

For synthesis based on a hydrazone strategy, see:. 
(d)M.T. Crimmins and S.W. Rafferty, Tetrahedron Lett. 37 (1996), pp. 5649-5652.

(e)D. Enders, W. Gatzweiler and E. Dederichs, Tetrahedron 46 (1990), pp. 4757-4792.

(f)S.L. Schreiber and Z. Wang, J. Am. Chem. Soc. 107 (1985), pp. 5303-5305.

For an account of natural product synthesis based on a dithiane strategy see:.

(g)A.B. Smith III and C.M. Adams, Acc. Chem. Res. 37 (2004), pp. 365-377 and references cited therein.

(h)A.B. Smith III, A. Doughty, C. Sfouggatakis, C.S. Bennet, J. Koyanagi and M. Takeuchi, Org. Lett. 4 (2002), pp. $783-786$.

8 (a)J.Y.C. Chan, L. Hough and A.C. Richardson, J. Chem. Soc., Chem. Commun. (1982), pp. 1151-1153.

(b)J.Y.C. Chan, L. Hough and A.C. Richardson, J. Chem. Soc., Perkin Trans. 1 (1985), pp. 1457-1462. (c)G. Jeminet, M.

Lemaire, J.G. Gourcy and F. Posada, Synthesis (1995), pp. 627-629.

9 M. Goubert, I. Canet and M.E. Sinibaldi, Eur. J. Org. Chem. (2006), pp. 4805-4812.

10 M. Goubert, L. Toupet, M.E. Sinibaldi and I. Canet, Tetrahedron 63 (2007), pp. 8255-8266.

11 G.T. Teregulova, L.Z. Rol'nik, S.S. Zlot-skii, D.L. Rakhmankulov, N.N. Silishchev, T.A. Sokolova and R.Kh. Khazipov, J. Appl. Chem. USSR 1 (1989), pp. 1501-1504.

12 The methylene group in the $\alpha$ position of the benzyloxime function is shielded when placed in a cis position relative to the benzyloxy group see: T. Naito, K. Nakagawa, T. Nakamura, A. Kasei, I. Ninomiya and T. Kiguchi, J. Org. Chem. 64 (1999), pp. 2003-2009.

13

(a) Challis, B. C.; Challis, J. A. In The Chemistry of Functional Groups: Reaction of the Carboxamide Group; Pataï, S., Ed.; London, 1970; Chapter 13;

(b)V.M. Mićović and M.L.J. Mihailović, J. Org. Chem. 18 (1953), pp. 1190-1200. 